2013

\title{
H I Absorption Toward H Ii Regions At Small Galactic Longitudes
}

C. Jones

J.M. Dickey

J.R. Dawson

N. M. McClure-Griffiths

L. D. Anderson

See next page for additional authors

Follow this and additional works at: https://researchrepository.wvu.edu/faculty_publications

\section{Digital Commons Citation}

Jones, C.; Dickey, J. M.; Dawson, J. R.; McClure-Griffiths, N. M.; Anderson, L. D.; and Bania, T. M., "H I Absorption Toward H Ii Regions At Small Galactic Longitudes" (2013). Faculty Scholarship. 550.

https://researchrepository.wvu.edu/faculty_publications/550 
Authors

C. Jones, J. M. Dickey, J. R. Dawson, N. M. McClure-Griffiths, L. D. Anderson, and T. M. Bania 


\title{
H I ABSORPTION TOWARD H II REGIONS AT SMALL GALACTIC LONGITUDES
}

\author{
C. Jones ${ }^{1}$, J. M. Dickey ${ }^{1}$, J. R. Dawson ${ }^{1,2}$, N. M. McClure-Griffiths ${ }^{2}$, L. D. Anderson ${ }^{3}$, and T. M. Bania ${ }^{4}$ \\ ${ }^{1}$ School of Mathematics and Physics, Private Bag 37, University of Tasmania, Hobart 7001, Australia \\ ${ }^{2}$ CSIRO Astronomy and Space Science, ATNF, P.O. Box 76, Epping, NSW 1710, Australia \\ ${ }^{3}$ Department of Physics, West Virginia University, Morgantown, WV 26506, USA \\ ${ }^{4}$ Institute for Astrophysical Research, Department of Astronomy, Boston University, 725 Commonwealth Avenue, Boston, MA 02215, USA \\ Received 2013 May 7; accepted 2013 July 16; published 2013 August 22
}

\begin{abstract}
We make a comprehensive study of $\mathrm{H}$ I absorption toward $\mathrm{H}$ II regions located within $|l|<10^{\circ}$. Structures in the extreme inner Galaxy are traced using the longitude-velocity space distribution of this absorption. We find significant H I absorption associated with the Near and Far 3 kpc Arms, the Connecting Arm, Bania's Clump 1, and the $\mathrm{H}$ I Tilted Disk. We also constrain the line-of-sight distances to $\mathrm{H}$ II regions, by using $\mathrm{H}$ I absorption spectra together with the $\mathrm{H}$ II region velocities measured by radio recombination lines.
\end{abstract}

Key words: Galaxy: structure - H II regions

Online-only material: color figures, figure set

\section{INTRODUCTION}

The extreme inner Galaxy (EIG) has long been the subject of intense astrophysical study as it provides excellent opportunities to explore dynamics, phenomena (from stellar to galactic scales), and physical environments which do not exist in the large-scale Galactic disk.

Throughout this paper, we refer to the area inside of, and including, the $3 \mathrm{kpc}$ Arms as the EIG (i.e., $R_{\text {Gal }} \lesssim 4 \mathrm{kpc}$ ). "Inner Galaxy" is a term already used to describe the areas of the Milky Way inside the solar circle, likewise the term "Galactic center" (GC) usually refers to the relatively small area with a Galactocentric radius less than a few hundred parsecs.

Useful reviews of the EIG environment include Morris \& Serabyn (1996) and Blitz et al. (1993), who discuss the interstellar medium (ISM) and structural components, respectively.

Radio observations of the EIG region have been performed since the 1950s (using the Dwingeloo $26 \mathrm{~m}$ antenna; van Woerden et al. 1957). These early studies discovered largescale H I features with non-circular motions (Oort 1977), and concentrated on understanding these individual structures, or particular objects.

The EIG has been extensively observed in CO. Molecular tracers probe denser material than neutral hydrogen $(\mathrm{HI})$ and $\mathrm{CO}$ is readily observed, therefore $\mathrm{CO}$ observations allow for analysis of regions in which the ISM is concentrated into structures such as arms and bars (Dame et al. 2001). In contrast, observations of atomic gas trace diffuse interstellar clouds.

While HI in the EIG has been well studied at low resolutions, it is only recently that high-resolution $\mathrm{HI}$ data which cover the entire EIG region have become available (i.e., ATCA H I Galactic Center Survey (HIGCS); McClure-Griffiths et al. 2012). These high-resolution H I data allow an analysis of the beginnings of the spiral arms; the transition between orbits associated with the bar; a comparison to high-resolution molecular observations, dynamical models, and molecular transitions; as well as investigations into the association of $\mathrm{H}$ I with the Galactic wind (McClure-Griffiths et al. 2012).

As a result of the variation in the temperature of interstellar hydrogen, H I emission and absorption spectra probe different phases of the ISM. In most emission spectra, it is the warmer components that dominate. However, cool gas is readily observed in absorption against background continuum sources, where it may be disentangled from warmer material along the line of sight. One advantage to studying $\mathrm{H}$ I absorption in the EIG is that it probes this predominantly cool material, which tends to be more localized in space, and more closely confined to structural entities such as arms.

Previous H I absorption studies have been vital to our understanding of the structure, rotation, and the nature of atomic gas in the EIG region. These include observations of absorption features associated with non-circular velocities, Radio Arc nonthermal filaments, as well as particular objects including SgrA* (Lang et al. 2010 and references therein).

While high-resolution $\mathrm{HI}_{\mathrm{I}}$ absorption measurements have been made toward several bright, or otherwise interesting, EIG continuum sources (Uchida et al. 1992; Roy 2003; Lang et al. 2010 and references therein) a complete H I absorption study of the EIG region has not been attempted. This present $\mathrm{HI}$ absorption survey constitutes the most complete study of $\mathrm{H} \mathrm{I}$ absorption against the continuum emission from the entire sample of $\mathrm{H}$ II regions known with $|l|<10^{\circ}$. This study is only possible due to recent $\mathrm{H}$ II region discovery surveys (which provide a list of target continuum regions with which to measure absorption against) and improved resolution in $\mathrm{H}$ I surveys that include the GC region.

In addition to providing a sample of bright continuum sources against which to measure $\mathrm{HI}_{\mathrm{I}}$ absorption, $\mathrm{H}_{\mathrm{II}}$ regions also provide an important secondary tracer of Galactic structure: the $\mathrm{H}$ II regions themselves. Galactic $\mathrm{H}$ II regions are the formation sites of massive OB stars, which have a main-sequence lifetime of $\sim$ tens of millions of years. As a result, Galactic H II regions reveal the locations of current massive star formation, indicate the present state of the ISM, provide a unique probe of Galactic chemical evolution, and are the archetypical tracers of Galactic spiral structure (Anderson et al. 2011).

In this work, we measure $\mathrm{H}$ I absorption against only those $\mathrm{H}$ II regions with known radio recombination line (RRL) velocities. This sub-sample is discussed in Section 2, and the method of $\mathrm{HI}$ absorption is described in Section 3.

We then summarize the known EIG structures (Section 4.1) and their locations in longitude-velocity $(l v)$ space. We plot 
these structures on an " $l v$ crayon diagram," and use the diagram to consider the EIG $l v$ distribution of $\mathrm{H}$ I absorption, in Section 5, and later for $\mathrm{H}$ II regions (Section 6).

We combine the results from Sections 5 and 6 to explore the Galactic distribution of $\mathrm{H}$ II regions (Section 7) - through determining the lower limit of the line-of-sight distance to each $\mathrm{H}$ II region based on its $\mathrm{HI}_{\mathrm{I}}$ absorption profile and systemic velocity.

Finally, a discussion of individual sources appears in the Appendix.

\section{DATA AND SOURCE SELECTION}

Large-scale, high-resolution astronomical surveys are now publicly available in many wavelength regimes. This work uses large-scale $\mathrm{H}$ i data and radio continuum maps.

\subsection{Radio Continuum}

Radio continuum maps were sourced from the NRAO VLA Sky Survey (NVSS; Condon et al. 1998) and the Southern Galactic Plane Survey (SGPS; McClure-Griffiths et al. 2005).

The NVSS covers $82 \%$ of the sky (north of $\delta=-40^{\circ}$ ) at $1.4 \mathrm{GHz}$, resulting in $23264^{\circ} \times 4^{\circ}$ continuum cubes of Stokes parameters and a catalog of continuum emission sources. Only the Stokes I maps were used for this work. It should be noted that the NVSS maps do not include zero spacing $(u, v)$ information and therefore many larger diffuse emission regions, particularly those in the Lockman et al. (1996) catalog, are not detected.

\subsection{Neutral Hydrogen, $H_{I}$}

For this work, Hi absorption spectra were extracted from the two SGPS data sets $\left(5^{\circ}<|l|<10^{\circ}\right)$ and the ATCA HIGCS $\left(5^{\circ}<|l|\right.$; McClure-Griffiths et al. 2012). Observations for the SGPS (I and II) and ATCA HIGCS were performed with the Australia Telescope Compact Array (ATCA) and supplemented with data from the Parkes Radio Telescope. The three surveys provide continuous coverage of the inner Galactic plane $\left(253^{\circ}<l<20^{\circ}\right)$ at $\sim 2^{\prime}$ resolution.

\subsection{Radio Recombination Lines}

Catalogs of RRLs provide systemic velocities for $\mathrm{H}$ II regions. Large-scale surveys of RRLs from $\mathrm{H}$ in regions were performed during the 1960s to 1980s. More recently, the Green Bank Telescope HiI Region Discovery Survey (GBTHRDS; Anderson et al. 2011) covered $343^{\circ}<l<67^{\circ}$ and detected RRLs from 448 new $\mathrm{H}$ II regions, effectively doubling the number known in that longitude range. The GBTHRDS is complete to $180 \mathrm{mJy}$ at $9 \mathrm{GHz}$, and is able to detect all $\mathrm{H}$ II regions ionized by a single O star to a distance of $12 \mathrm{kpc}$.

In addition, the GBTHRDS also includes a catalog of known $\mathrm{H}$ II regions as of 2010 . For the $|l|<10^{\circ}$ region, this includes the combined works of Downes et al. (1980), Wink et al. (1982), Caswell \& Haynes (1987), Lockman (1989), Lockman et al. (1996), and Sewilo et al. (2004). The GBTHRDS team carefully compiled this "known" catalog, removing duplicate sources through radio continuum and mid-infrared inspection. However, they note that it is "likely to contain some residual contamination and duplicate entries." The combination of this "known" catalog of H II regions and the GBTHRDS source list, within $|l|<10^{\circ}$, provided the sample list of regions for this work. Both the GBTHRDS catalog and the compilation of previous catalogs can be found at http://www.cv.nrao.edu/hrds/.
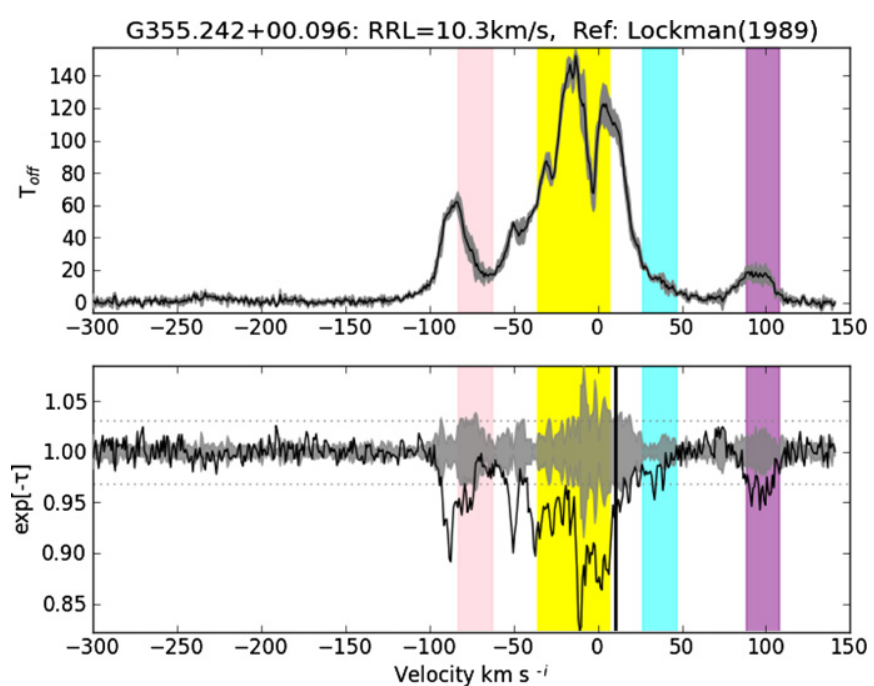

Figure 1. H I emission/absorption spectrum pairs. In each figure, the top panel shows the emission spectra. The emission is shown by the solid line (this is the average of the three "off" positions, see Section 3.1) and the emission envelope (difference between the "off" positions) is shown in gray. Absorption, $e^{-\tau}$, is displayed in the bottom panel. The H I absorption spectrum (see Equation (2)) is shown by the solid line and the gray envelope signifies $3 \sigma_{e^{-\tau}}$ (calculated from the emission envelope, see Section 3). The absorption panel also displays the RRL velocities of the H II region (solid vertical lines) and the fluctuation in the baseline of the absorption spectrum $\left(3 \sigma_{\mathrm{rms}_{e}-\tau}\right)$ (horizontal dotted lines). The $\mathrm{H}$ II region name, RRL velocity, and reference are shown as well as the expected velocity ranges of EIG features (see Section 4.1) with the same color system as Figure 2.

(A color version and the complete figure set (151 images) of this figure are available in the online journal.)

\subsubsection{H II Regions Selected}

There are nearly 200 known $\mathrm{H}_{\text {II }}$ regions in the range $|l|<$ $10^{\circ},|b|<1.5$ with observed RRL velocities. H I absorption spectra were extracted toward a total of 151 of these $\mathrm{H}$ iI regions (see Figure 1). The remaining $\mathrm{H}$ II regions were either not visible in the NVSS continuum maps (also used by the GBTHRDS), usually diffuse H II regions from the Lockman et al. (1996) catalog, or $\mathrm{H}$ II regions with coordinates that could refer to several continuum sources-see Table 1 . Therefore, this study obtains $\mathrm{H}_{\mathrm{I}}$ absorption spectra toward over $80 \%$ of known $\mathrm{H}$ II regions with $|l|<10^{\circ}$. The "name" for each $\mathrm{H}$ II region is taken from the RRL catalog from which it was sourced.

\section{EXTRACTION OF THE H I ABSORPTION SPECTRA}

The hyperfine transition that creates the $21 \mathrm{~cm} \mathrm{H}$ I line is often seen in both emission and absorption from the same region-indeed for most continuum sources a mixture of emission and absorption is observed. Therefore, a method is required to separate the two.

\subsection{Emission/Absorption Method}

The emission/absorption method (described in detail by Kolpak et al. 2003) compares foreground cloud absorption with continuum emission from a background target. Absorption, $e^{-\tau}$, is derived by comparing the brightness temperature as a function of velocity $(v)$ both on $\left(T_{\mathrm{on}}\right)$ and off (i.e., the emission spectrum, $\left.T_{\text {off }}\right)$ the continuum source. Continuum maps were inspected with the KARMA package (Gooch 1996) to ascertain the pixel positions for "on" and "off" spectra to be extracted from the H I cubes; one "on" and three "off" source positions were chosen 
Table 1

H II Regions That Were Not Included in This Work

\begin{tabular}{|c|c|c|}
\hline H II Region & Reference & Note \\
\hline G351.265 + 01.019 & GBTHRDS (2011) & NV \\
\hline $\mathrm{G} 351.590+00.183$ & Lockman (1989) & MS \\
\hline $\mathrm{G} 353.035+00.748$ & Lockman (1989) & MS \\
\hline $\mathrm{G} 353.083+00.358$ & Lockman (1989) & MS \\
\hline G357.998-00.159 & Lockman (1989) & $\mathrm{DC}$ \\
\hline G358.319-00.414 & Lockman et al. (1996) & NV \\
\hline G358.623-00.066 & Caswell \& Haynes (1987) & $\mathrm{DC}$ \\
\hline G358.661-00.575 & Lockman et al. (1996) & NV \\
\hline G358.664-00.575 & Lockman et al. (1996) & NV \\
\hline G358.974-00.021 & Lockman et al. (1996) & $\mathrm{NV}$ \\
\hline G359.186-00.026 & Caswell \& Haynes (1987) & $\mathrm{DC}$ \\
\hline G359.730-00.407 & Downes et al. (1980) & NV \\
\hline $\mathrm{G} 359.783+00.040$ & GBTHRDS (2011) & NV \\
\hline G359.929+00.045 & GBTHRDS (2011) & NV \\
\hline G000.394-00.540 & Downes et al. (1980) & NV \\
\hline G000.521 + 00.178 & Lockman et al. (1996) & $\mathrm{NV}$ \\
\hline G000.605 + 00.325 & Lockman et al. (1996) & NV \\
\hline G000.656-00.058 & Downes et al. (1980) & NV \\
\hline G000.829+00.193 & Downes et al. (1980) & NV \\
\hline $\mathrm{G} 001.323+00.086$ & Caswell \& Haynes (1987) & $\mathrm{DC}$ \\
\hline $\mathrm{G} 002.303+00.243$ & Lockman (1989) & MS \\
\hline G005.049+00.254 & Lockman (1989) & NV \\
\hline G005.332+00.081 & Lockman et al. (1996) & MS \\
\hline G006.616-00.545 & Lockman et al. (1996) & NV \\
\hline G006.667-00.247 & Lockman (1989) & NV \\
\hline G006.979-00.250 & Lockman (1989) & NV \\
\hline G007.002-00.015 & Lockman et al. (1996) & NV \\
\hline G007.299-00.116 & Lockman (1989) & NV \\
\hline $\mathrm{G} 007.387+00.668$ & Lockman (1989) & NV \\
\hline G008.415 + 00.033 & Lockman et al. (1996) & NV \\
\hline G008.786-00.034 & Lockman et al. (1996) & NV \\
\hline G009.176 + 00.032 & Lockman et al. (1996) & $\mathrm{DC}$ \\
\hline
\end{tabular}

Notes. NV: not visible at the SGPS pixel scale; MS: many continuum sources present at this location; DC: duplicate (in both catalogs).

in accordance with the criteria identified in Jones \& Dickey (2012).

The simplest radiative transfer situation gives

$$
T_{\mathrm{on}}(v)=\left(T_{\mathrm{bg}}+T_{\text {cont }}\right) e^{-\tau}(v)+T_{S}(v)\left(1-e^{-\tau(v)}\right),
$$

where $T_{\text {cont }}$ is the continuum source brightness temperature, $T_{s}$ is the spin temperature of the foreground cloud, and $T_{\text {bg }}$ represents any other background contribution. Assuming that both the "on" and "off" spectra sample the same gas, subtraction of one from the other removes the common $T_{s}(v)\left[1-e^{-\tau}(v)\right]$ and $T_{\mathrm{bg}}$ terms allowing optical depth to be measured directly. The absorption is then given by

$$
e^{-\tau}=\frac{T_{\text {on }}-T_{\text {off }}}{T_{\text {cont }}} .
$$

The quality of an absorption spectrum is not determined by radiometer noise, but rather the accuracy of estimating the emission both on and around the background continuum source. As such, we require absorption to be significant in relation to both emission fluctuations and the noise in the baseline of the absorption spectra (in a region without absorption features).

For each absorption spectrum, velocity channels with significant absorption were selected for analysis. Significant absorption is defined to satisfy both

1. significance at the $3 \sigma_{e^{-\tau}}$ level, where $\sigma_{e^{-\tau}}$ is calculated from the emission fluctuation envelope (the difference in emission between "off" sources), and
2. significance at the $3 \sigma_{\mathrm{rms}_{e^{-\tau}}}$ level, where $\sigma_{\mathrm{rms}_{e}-\tau}$ is the fluctuation in the baseline of the absorption spectrum.

The NVSS continuum maps are biased toward smaller continuum temperatures (see Section 2.1) as they do not include all diffuse continuum emission. However, as $T_{\text {cont }}$ acts as a scaling factor for $e^{-\tau}$ (see Equation (2)), $\sigma_{e^{-\tau}}$ and $\sigma_{\mathrm{rms}_{e^{-\tau}}}$ will also scale proportionately with any change in continuum temperature.

Emission and absorption spectrum pairs toward each $\mathrm{H}$ II region appear in Figure 1.

\section{LONGITUDE-VELOCITY OVERVIEW OF THE EXTREME INNER GALAXY}

Absorption spectra along lines of sight through the Galactic disk within the longitude range $|l|<10^{\circ}$ are complex and difficult to interpret. This longitude region includes structures associated with the GC and EIG ( $R_{\mathrm{Gal}} \lesssim 4 \mathrm{kpc}$ ), with highly non-circular motions; as well as features with velocities consistent with circular disk rotation $\left(R_{\mathrm{Gal}} \gtrsim 4 \mathrm{kpc}\right)$.

Here we use the summary of EIG structures (Section 4.1) to construct an " $l v$ crayon plot" (Figure 2), marking prominent EIG features based on the integrated intensity of ${ }^{12} \mathrm{CO}$ in the same $l, b$ range (from Dame et al. 2001).

$\mathrm{CO}$ traces denser material than $\mathrm{HI}$ and therefore picks out the densest features. In the inner Galaxy, atomic gas often acts to shield associated regions of molecular gas from photodissociation (Dickey \& Lockman 1990). Therefore, H I absorption features may be identified with known EIG molecular emission features using correlations in velocity structure (Lang et al. 2010).

As a result, this plot provides a useful reference, which we use to consider the $l v$ distribution of $\mathrm{H}$ I absorption; Section 5 (and later $\mathrm{H}$ II region RRL velocities; Section 6).

\subsection{Structures in the Extreme Inner Galaxy}

Structures in the EIG include a long, thin bar, a shorter, boxy-bulge bar, the Near and Far $3 \mathrm{kpc}$ Arms, tilted Hi inner disk or ring, central molecular zone, and thin twisted $100 \mathrm{pc}$ ring (McClure-Griffiths et al. 2012). In addition to these more prominent structures, recent $l v$ diagrams from $\mathrm{H}$ i and $\mathrm{CO}$ observations show many "clumpy" sub-structures, not seen in previous EIG models (Baba et al. 2010).

The angular extent of some of these EIG features is quite large: the Near $3 \mathrm{kpc}$ Arm is observed to $l<348^{\circ}$, and to surround all $\mathrm{HI}_{\mathrm{I}}$ emission associated with the EIG region, a latitude range of at least $|b| \leqslant 8^{\circ}$ is required (Burton \& Liszt 1983)—well beyond the range of known $\mathrm{H}$ II regions $\left(|b|<\sim 2^{\circ}\right)$.

Many of these features are not often explicitly discussed in the literature and precise distances are usually unknown (Fux 1999). A summary of the EIG gas structures, many of which are visible in $\mathrm{H}$ I absorption spectra, appears below. Often these objects have several names in the literature, or several distinct features have been given the same name by different authors.

For a discussion of the evolution of the understanding of $\mathrm{H}_{\mathrm{I}}$ and CO $l v$ models in the EIG, see Baba et al. (2010). Burton $\&$ Liszt (1983) provide a series of $l v$ diagrams with prominent features identified.

\subsubsection{Near and Far $3 \mathrm{kpc}$ Arms}

Near $3 \mathrm{kpc}$ Arm. The Near $3 \mathrm{kpc}$ Arm or Expanding $3 \mathrm{kpc}$ Arm was discovered in the late 1950s and is known to lie in front of the GC (van Woerden et al. 1957). However, it is not yet 

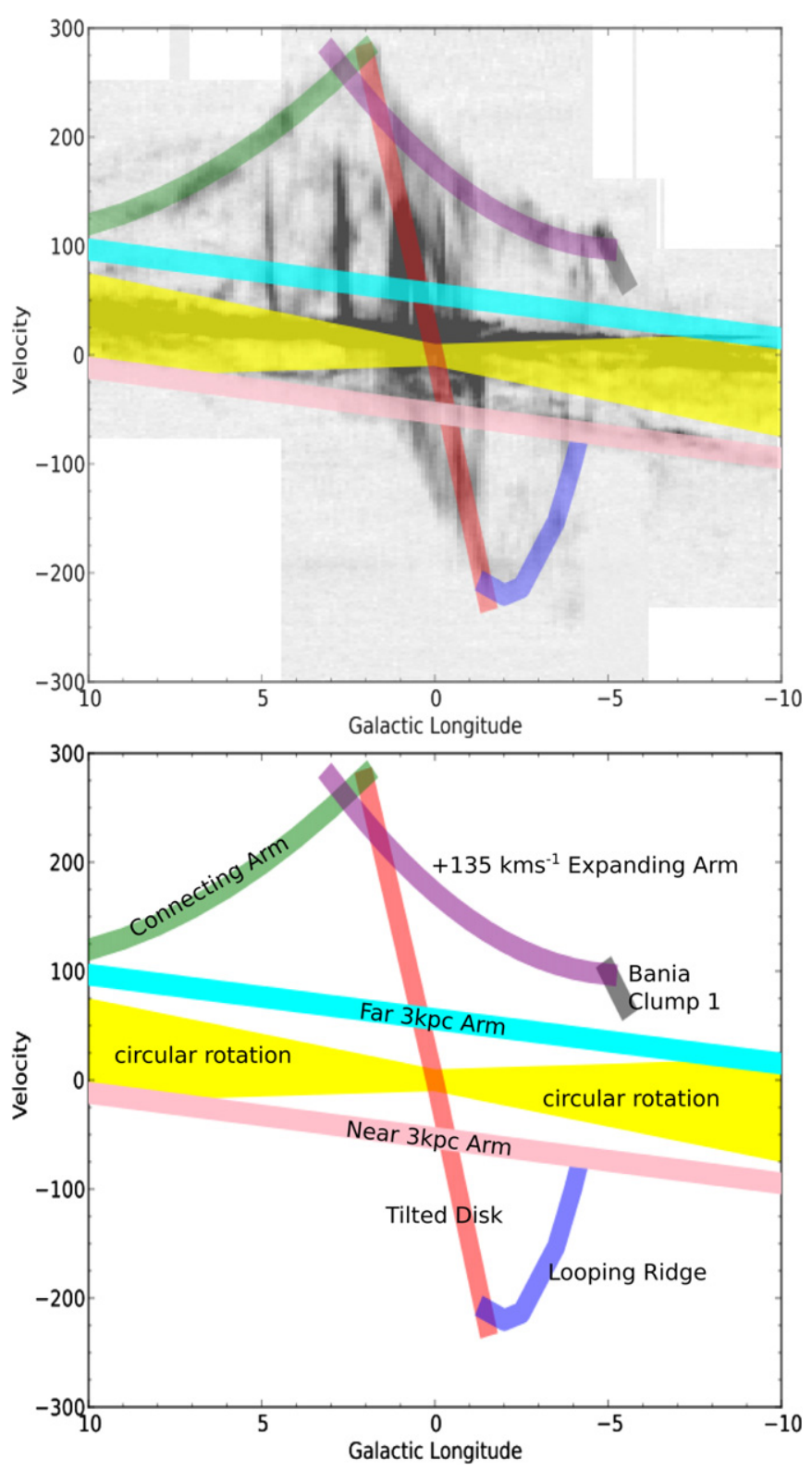

Figure 2. Longitude-velocity "crayon" diagram for $l<\left|10^{\circ}\right|, b<|1.5|^{\circ}$. Top panel: the "crayon" features overlaid on CO emission map (Dame et al. 2001). Bottom panel: the "crayon" features (each with a velocity width of $20 \mathrm{~km} \mathrm{~s}^{-1}$ ). The "crayon" color system is as follows: yellow, circular rotation allowed velocity envelope; green, Connecting Arm; purple, $+135 \mathrm{~km} \mathrm{~s}^{-1}$ Expanding Arm; gray, Bania's Clump 1; red, Tilted Disk; cyan, Far 3 kpc Arm; yellow, velocities allowed by circular disk rotation; pink,Near $3 \mathrm{kpc}$ Arm; blue, Looping Ridge. While not explicitly labeled in the crayon diagram, Bania's Clump 2 can be seen as the thick vertical CO feature at $l \sim 3^{\circ}, 0 \lesssim v \lesssim 200 \mathrm{~km} \mathrm{~s}^{-1}$ in the top panel.

(A color version of this figure is available in the online journal.)

agreed whether the Near $3 \mathrm{kpc}$ Arm is a lateral arm surrounding the bar, or a small arm extending from the end of the bar, or an arm located where the bar meets its corotation radius (Rodríguez-Fernández 2011). Stretching over $35^{\circ}$ in longitude, the Near $3 \mathrm{kpc}$ Arm exists at "forbidden" velocities and its discovery provided vital early support for a Galactic bar (Dame $\&$ Thaddeus 2008, and references therein). The Near $3 \mathrm{kpc}$ Arm appears as the pink line in Figure 2.

Far $3 \mathrm{kpc}$ Arm. Despite the tendency for major anomalous velocity features in the GC to occur in positive and negative velocity pairs (Burton \& Liszt 1983), it was originally thought that there was no far-side counterpart to the Near $3 \mathrm{kpc}$ Arm (Dame \& Thaddeus 2008 and references therein). Fux (1999) supposed the $135 \mathrm{~km} \mathrm{~s}^{-1}$ Arm (discussed below) was the feature symmetric to the Near $3 \mathrm{kpc}$ Arm-however, Fux also noted compositional differences between the $+135 \mathrm{~km} \mathrm{~s}^{-1}$ and Near $3 \mathrm{kpc}$ Arm, attributing these to an asymmetric spiral structure. Dame \& Thaddeus (2008) reported the detection (in CO and then followed up in $\mathrm{HI}$ ) of the far-side counterpart to the Near $3 \mathrm{kpc}$ Arm, named the Far $3 \mathrm{kpc}$ Arm. The Far $3 \mathrm{kpc}$ Arm appears as the cyan line in Figure 2.

\subsubsection{H I Tilted/Nuclear Disk}

The H I inner Tilted Disk, proposed by Liszt \& Burton (1980), was the result of a full three-dimensional analysis of all known Hi emission in the inner kiloparsec of the Galaxy. It was modeled by a series of closed elliptical gas orbits. The disk is oriented at 23.7 with respect to the Galactic plane and accounts for positive velocity $\mathrm{HI}$ emission at $b<0^{\circ}, l>0^{\circ}$ and negative velocity gas at $b>0^{\circ}, l<0^{\circ}$ (HIGCS). In Figure 2, the Tilted Disk appears as the red line crossing through $(l, v)=(0,0)$.

\subsubsection{The Expanding Arm(s)}

$+135 \mathrm{~km} \mathrm{~s}^{-1}$ Arm. The location of the $+135 \mathrm{~km} \mathrm{~s}^{-1}$ Arm, or Expanding Arm, is contentious throughout the literature: Fux (1999) assumes it is the far-side counterpart to the Near $3 \mathrm{kpc}$ Arm (see Section 4.1.1), Bania (1980) argues that the $3 \mathrm{kpc}$ and $+135 \mathrm{~km} \mathrm{~s}^{-1}$ Arms cannot be described together as a kinematic ring, and Baba et al. (2010) model the $+135 \mathrm{~km} \mathrm{~s}^{-1}$ Arm as part of the end of the bar on the far side.

The $+135 \mathrm{~km} \mathrm{~s}^{-1}$ Arm is more clumpy than the Near $3 \mathrm{kpc}$ Arm (Fux 1999) and extends nearly $30^{\circ}$ in longitude and spans $3^{\circ}$ in latitude near the $\mathrm{GC}\left(-1^{\circ}<b<2^{\circ}\right.$ at $l=359^{\circ}$; Uchida et al. 1992). Distance estimates for the $+135 \mathrm{~km} \mathrm{~s}^{-1}$ Arm vary; Simonson \& Madder (1973) and Bania (1980) give galactocentric radii only ( $3.4 \mathrm{kpc}$ and $2.8-3.5 \mathrm{kpc}$, respectively), whereas Uchida et al. (1992) give a distance estimate of about $2 \mathrm{kpc}$ behind the GC (i.e., $D_{\mathrm{los}}>10 \mathrm{kpc}$ ).

In Figure 2, the $+135 \mathrm{~km} \mathrm{~s}^{-1}$ Expanding Arm appears as the purple curve.

Bania's Clumps. The individual emission clumps that comprise the $+135 \mathrm{~km} \mathrm{~s}^{-1}$ Arm probably either include Bania's Clumps 1 and 2 (Bania 1980; Bania et al. 1986), or the two molecular cloud complexes are entering the dust lane shock (Liszt 2008). A detailed discussion of the $\mathrm{H}$ I properties of Bania's Clump 2 can be found in McClure-Griffiths et al. (2012).

Bania's Clump 1 is seen as the gray line in Figure 2, whereas Bania's Clump 2 is seen as the thick vertical $\mathrm{CO}$ feature at $l \approx 3^{\circ}, \sim 0<v<\sim 200 \mathrm{~km} \mathrm{~s}^{-1}$ in the $\mathrm{CO}$ emission map (top panel of Figure 2).

$-135 \mathrm{~km} \mathrm{~s}^{-1}$ Feature. Just as the Near $3 \mathrm{kpc}$ Arm has a nearly symmetrical velocity and spatial counterpart a $-135 \mathrm{~km} \mathrm{~s}^{-1}$ Feature is thought to be located in the foreground of the GC, but behind the Near $3 \mathrm{kpc}$ Arm, as it is seen in $\mathrm{OH}$ absorption (Uchida et al. 1992). This feature is much less distinct than the $+135 \mathrm{~km} \mathrm{~s}^{-1}$ Arm, indeed Bania (1980) did not detect it. This feature is not included in Figure 2.

\subsubsection{Connecting Arm and Looping Ridge}

Two features - the Connecting Arm and Looping Ridge-are visible in $\mathrm{CO}$ and $\mathrm{HI}$ emission, as well as in near-infrared dust extinction (Marshall et al. 2008 and HIGCS). These features lead the bar major axis and are the location of strong shearing shocks, resulting in high velocities (Fux 1999). 
The extent of both structures in $l, b, v$ has been explored in detail by Marshall et al. (2008) who use CO data to localize emission to specific $l v$ structures.

Connecting Arm-positive velocity feature. The Connecting Arm (at extreme positive velocities and longitudes) was named as it seems to link the nuclear ring/disk to the outer disk (Fux 1999). The arm was sufficiently prominent in $\mathrm{H}$ i to be described as a distinct feature in early EIG surveys (Liszt 2008). The location of the Connecting Arm, in front of or behind the GC, was originally unclear; it has been interpreted as part of the central bar on the far side of the GC, or as an artifact due to velocity crowding along the line of sight, but it is now accepted to be a near-side dust lane (Fux 1999 and references therein).

The Connecting Arm appears as the green curve in Figure 2.

Looping Ridge-negative velocity feature. The corresponding feature to the Connecting Arm (at negative velocities and longitudes) is not always treated as a distinct feature (Liszt 2008) and remains unnamed, however, McClure-Griffiths et al. (2012) refer to the negative feature as the "Looping" Ridge. Liszt (2008) suggests that the Looping Ridge may be (temporarily) starved of gas and hence more difficult to detect and analyze.

In Figure 2, the Looping Ridge appears as the blue curve.

\section{LONGITUDE-VELOCITY DISTRIBUTION OF H I ABSORPTION TOWARD THE EXTREME INNER GALAXY}

Figure 3 displays the $\mathrm{H}$ I absorption in $l v$ space, and compares this distribution with the EIG structures (Section 4.1), H I and $\mathrm{CO}$ emission.

Table 2 notes if significant $\mathrm{H}$ I absorption is associated with any EIG feature for each $\mathrm{H}$ iI region.

It is immediately obvious that the $\mathrm{H}$ I absorption distribution is not random, but closely follows the identified EIG features. This is not surprising as cold $\mathrm{H}_{\mathrm{I}}$ gas, seen in absorption, is a good tracer of Galactic structure.

$\mathrm{H}$ I absorption is associated with the allowed circular rotation velocities (as expected) as well as the Near and Far 3 kpc Arms, Connecting Arm, and Bania's Clump 1.

\subsection{H I Absorption Associated with the $3 \mathrm{kpc}$ Arms}

The CO emission from both the Near and Far 3 kpc Arms is contained within $|b|<1^{\circ}$ (Dame \& Thaddeus 2008), similar to the Galactic latitude range of the $\mathrm{H}$ II regions in this work $(|b| \lesssim 1.5)$. Furthermore, both $3 \mathrm{kpc}$ Arms are thought to span $|l| \lesssim 13^{\circ}$, which includes the whole longitude range of this work. Therefore, if an $\mathrm{H}$ II region is located behind either of the $3 \mathrm{kpc}$ Arms, H I absorption should be visible at velocities pertaining to that arm.

Figure 3 demonstrates that significant $\mathrm{H}$ I absorption is seen toward the Near $3 \mathrm{kpc}$ Arm at all longitudes; although there is a conspicuous gap in absorption at longitudes $\sim 356<l^{\circ}<\sim$ 358 , consistent with a paucity of $\mathrm{H}$ II regions for which to measure absorption toward. Indeed, $67 \% \mathrm{H}$ II regions display absorption associated with the Near $3 \mathrm{kpc}$ Arm.

There is less absorption associated with the Far $3 \mathrm{kpc}$ Arm than with the Near $(\sim 1.0: 3.3)$, with the site of greatest absorption for the Far $3 \mathrm{kpc}$ Arm centered at $l \approx 7^{\circ}$ (see Figure 4). The disparity in the amount of H I absorption may be an effect of the smaller latitude extent of the Far $3 \mathrm{kpc}$ Arm, which is particularly thin in the fourth quadrant (Dame \& Thaddeus 2008).
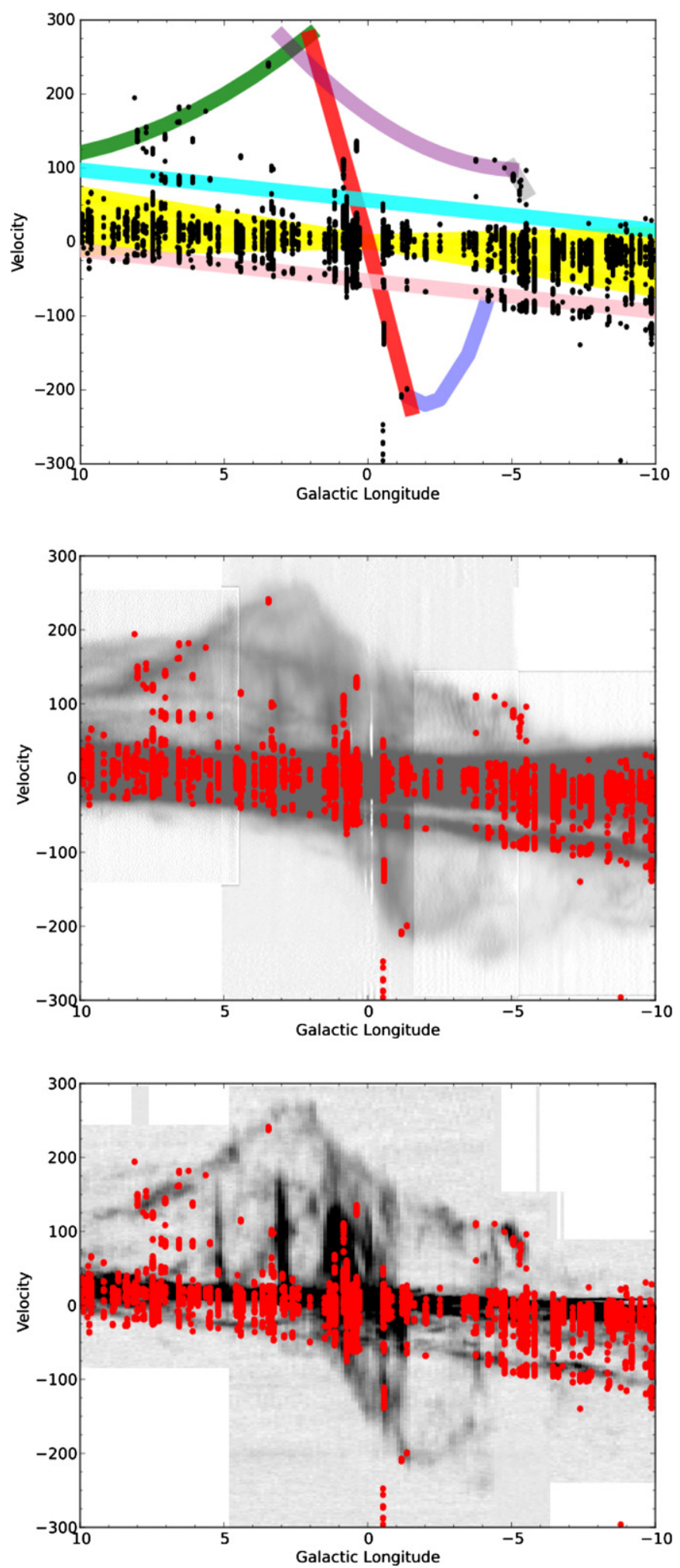

Figure 3. $l v$ diagrams showing velocity channels with significant absorption for each $\mathrm{H}$ II region overlaid onto: top panel: the "crayon" plot of EIG features (see Figure 2); middle panel: H I emission (the intensity map was created from the SGPS I and II and ATCA HIGCS); bottom panel: CO emission (see Figure 2).

(A color version of this figure is available in the online journal.)

Both the HIGCS and Dame \& Thaddeus (2008) report a bifurcation in the velocities Far $3 \mathrm{kpc}$ Arm (in $l v$ space) at $l<6^{\circ}$. There is limited evidence of this bifurcation at longitudes extending to $\approx 7^{\circ}$, the best example of this is in the absorption 
Table 2

Presence of Significant H I Absorption in EIG Features for Each H II Region

\begin{tabular}{|c|c|c|c|c|c|c|c|c|c|c|c|c|c|}
\hline Region & N3 & $\mathrm{CA}$ & $\mathrm{TD}$ & $+\mathrm{E} 135$ & $\mathrm{BC} 1$ & F3 & Region & N3 & $\mathrm{CA}$ & $\mathrm{TD}$ & E135 & $\mathrm{BC} 1$ & F3 \\
\hline G350.004+00.438 & $\mathrm{N}$ & $\ldots$ & $\ldots$ & $\cdots$ & $\ldots$ & $\mathrm{N}$ & G000.284-00.478 & $\mathrm{Y}$ & $\ldots$ & $\mathrm{N}$ & $\mathrm{N}$ & $\ldots$ & $\mathrm{N}$ \\
\hline $\mathrm{G} 350.129+00.088$ & $\mathrm{Y}$ & $\ldots$ & $\ldots$ & $\ldots$ & $\ldots$ & $\mathrm{N}$ & G000.361-00.780 & $\mathrm{Y}$ & $\ldots$ & $\mathrm{N}$ & $\mathrm{N}$ & $\ldots$ & $\mathrm{Y}$ \\
\hline G350.177+00.017 & $\mathrm{N}$ & $\cdots$ & $\cdots$ & $\cdots$ & $\cdots$ & $\mathrm{Y}$ & $\mathrm{G} 000.382+00.017$ & $\mathrm{Y}$ & $\cdots$ & $\mathrm{N}$ & $\mathrm{Y}$ & $\cdots$ & $\mathrm{N}$ \\
\hline $\mathrm{G} 350.330+00.157$ & $\mathrm{Y}$ & $\ldots$ & $\ldots$ & $\ldots$ & $\ldots$ & $\mathrm{Y}$ & G000.510-00.051 & $\mathrm{Y}$ & $\ldots$ & $\mathrm{N}$ & $\mathrm{N}$ & $\ldots$ & $\mathrm{N}$ \\
\hline $\mathrm{G} 350.335+00.107$ & $\mathrm{Y}$ & $\ldots$ & $\cdots$ & $\cdots$ & $\ldots$ & $\mathrm{N}$ & G000.572-00.628 & $\mathrm{Y}$ & $\ldots$ & $\mathrm{N}$ & $\mathrm{N}$ & $\ldots$ & $\mathrm{N}$ \\
\hline $\mathrm{G} 350.524+00.960$ & $\mathrm{~N}$ & $\ldots$ & $\cdots$ & $\ldots$ & $\ldots$ & $\mathrm{N}$ & G000.640+00.623 & $\mathrm{Y}$ & $\cdots$ & $\mathrm{Y}$ & $\mathrm{N}$ & $\cdots$ & $\mathrm{Y}$ \\
\hline G350.769-00.075 & $\mathrm{N}$ & $\ldots$ & $\ldots$ & $\ldots$ & $\ldots$ & $\mathrm{N}$ & G000.729-00.123 & $\mathrm{Y}$ & $\cdots$ & $\mathrm{N}$ & $\mathrm{N}$ & $\cdots$ & $\mathrm{Y}$ \\
\hline G350.813-00.019 & $\mathrm{N}$ & $\cdots$ & $\cdots$ & $\cdots$ & $\cdots$ & $\mathrm{Y}$ & G000.838+00.189 & $\mathrm{Y}$ & $\cdots$ & $\mathrm{Y}$ & $\mathrm{Y}$ & $\cdots$ & $\mathrm{Y}$ \\
\hline G350.996-00.557 & $\mathrm{N}$ & $\ldots$ & $\cdots$ & $\cdots$ & $\ldots$ & $\mathrm{Y}$ & G001.125-00.105 & $\mathrm{Y}$ & $\cdots$ & $\mathrm{N}$ & $\mathrm{Y}$ & $\cdots$ & $\mathrm{Y}$ \\
\hline $\mathrm{G} 351.028+00.155$ & $\mathrm{Y}$ & $\ldots$ & $\ldots$ & $\ldots$ & $\ldots$ & $\mathrm{Y}$ & G001.149-00.062 & $\mathrm{Y}$ & $\ldots$ & $\mathrm{Y}$ & $\mathrm{N}$ & $\ldots$ & $\mathrm{Y}$ \\
\hline G351.047-00.322 & $\mathrm{N}$ & $\ldots$ & $\ldots$ & $\ldots$ & $\ldots$ & $\mathrm{N}$ & G001.324 + 00.104 & $\mathrm{~N}$ & $\ldots$ & $\mathrm{N}$ & $\mathrm{N}$ & $\ldots$ & $\mathrm{N}$ \\
\hline $\mathrm{G} 351.192+00.708$ & $\mathrm{~N}$ & $\ldots$ & $\ldots$ & $\ldots$ & $\ldots$ & $\mathrm{N}$ & $\mathrm{G} 001.330+00.088$ & $\mathrm{Y}$ & $\ldots$ & $\mathrm{N}$ & $\mathrm{N}$ & $\ldots$ & $\mathrm{Y}$ \\
\hline $\mathrm{G} 351.201+00.483$ & $\mathrm{~N}$ & $\ldots$ & $\cdots$ & $\ldots$ & $\ldots$ & $\mathrm{N}$ & G001.488-00.199 & $\mathrm{Y}$ & $\ldots$ & $\mathrm{N}$ & $\mathrm{Y}$ & $\ldots$ & $\mathrm{Y}$ \\
\hline G351.358+00.666 & $\mathrm{N}$ & $\cdots$ & $\cdots$ & $\cdots$ & $\cdots$ & $\mathrm{Y}$ & G002.009-00.680 & $\mathrm{Y}$ & $\mathrm{N}$ & $\cdots$ & $\mathrm{N}$ & $\cdots$ & $\mathrm{N}$ \\
\hline $\mathrm{G} 351.359+01.014$ & $\mathrm{~N}$ & $\ldots$ & $\ldots$ & $\ldots$ & $\ldots$ & $\mathrm{N}$ & G002.404 + 00.068 & $\mathrm{~N}$ & $\mathrm{~N}$ & $\cdots$ & $\mathrm{N}$ & $\ldots$ & $\mathrm{N}$ \\
\hline G351.467-00.462 & $\mathrm{N}$ & $\ldots$ & $\ldots$ & $\ldots$ & $\ldots$ & $\mathrm{N}$ & G002.418-00.611 & $\mathrm{N}$ & $\mathrm{N}$ & $\ldots$ & $\mathrm{N}$ & $\ldots$ & $\mathrm{N}$ \\
\hline G351.601-00.348 & $\mathrm{N}$ & $\cdots$ & $\cdots$ & $\cdots$ & $\ldots$ & $\mathrm{Y}$ & G002.510-00.028 & $\mathrm{Y}$ & $\mathrm{N}$ & $\cdots$ & $\mathrm{Y}$ & $\cdots$ & $\mathrm{N}$ \\
\hline $\mathrm{G} 351.662+00.518$ & $\mathrm{Y}$ & $\ldots$ & $\cdots$ & $\cdots$ & $\ldots$ & $\mathrm{N}$ & G002.611 + 00.135 & $\mathrm{Y}$ & $\mathrm{N}$ & $\cdots$ & $\mathrm{N}$ & $\cdots$ & $\mathrm{N}$ \\
\hline G351.691+00.669 & $\mathrm{N}$ & $\cdots$ & $\cdots$ & $\cdots$ & $\cdots$ & $\mathrm{N}$ & G002.819-00.132 & $\mathrm{N}$ & $\mathrm{N}$ & $\cdots$ & $\mathrm{N}$ & $\cdots$ & $\mathrm{N}$ \\
\hline G352.233-00.151 & $\mathrm{Y}$ & $\ldots$ & $\ldots$ & $\ldots$ & $\ldots$ & $\mathrm{N}$ & G002.901-00.006 & $\mathrm{Y}$ & $\mathrm{N}$ & $\cdots$ & $\mathrm{N}$ & $\cdots$ & $\mathrm{N}$ \\
\hline G352.313-00.440 & $\mathrm{Y}$ & $\ldots$ & $\ldots$ & $\ldots$ & $\ldots$ & $\mathrm{Y}$ & G002.961-00.053 & $\mathrm{Y}$ & $\mathrm{N}$ & $\ldots$ & $\mathrm{N}$ & $\ldots$ & $\mathrm{Y}$ \\
\hline G352.398-00.057 & $\mathrm{Y}$ & $\ldots$ & $\ldots$ & $\ldots$ & $\ldots$ & $\mathrm{N}$ & G003.270-00.101 & $\mathrm{Y}$ & $\mathrm{N}$ & $\ldots$ & $\ldots$ & $\ldots$ & $\mathrm{Y}$ \\
\hline G352.521-00.144 & $\mathrm{Y}$ & $\ldots$ & $\ldots$ & $\ldots$ & $\ldots$ & $\mathrm{N}$ & G003.342-00.079 & $\mathrm{Y}$ & $\mathrm{Y}$ & $\ldots$ & $\cdots$ & $\ldots$ & $\mathrm{Y}$ \\
\hline $\mathrm{G} 352.610+00.177$ & $\mathrm{~N}$ & $\ldots$ & $\ldots$ & $\ldots$ & $\ldots$ & $\mathrm{N}$ & G003.439-00.349 & $\mathrm{Y}$ & $\mathrm{N}$ & $\ldots$ & $\ldots$ & $\ldots$ & $\mathrm{N}$ \\
\hline G352.611-00.172 & $\mathrm{Y}$ & $\cdots$ & $\cdots$ & $\cdots$ & $\cdots$ & $\mathrm{N}$ & G003.449-00.647 & $\mathrm{Y}$ & $\mathrm{Y}$ & $\cdots$ & $\cdots$ & $\cdots$ & $\mathrm{N}$ \\
\hline G352.866-00.199 & $\mathrm{Y}$ & $\cdots$ & $\cdots$ & $\cdots$ & $\cdots$ & $\mathrm{N}$ & G003.655-00.111 & $\mathrm{Y}$ & $\mathrm{N}$ & $\cdots$ & $\cdots$ & $\cdots$ & $\mathrm{N}$ \\
\hline G353.186+00.887 & $\mathrm{N}$ & $\ldots$ & $\ldots$ & $\ldots$ & $\ldots$ & $\mathrm{N}$ & G003.928-00.116 & $\mathrm{Y}$ & $\mathrm{N}$ & $\cdots$ & $\cdots$ & $\ldots$ & $\mathrm{Y}$ \\
\hline G353.218-00.249 & $\mathrm{Y}$ & $\ldots$ & $\cdots$ & $\ldots$ & $\ldots$ & $\mathrm{N}$ & G003.949-00.100 & $\mathrm{Y}$ & $\mathrm{N}$ & $\cdots$ & $\cdots$ & $\ldots$ & $\mathrm{N}$ \\
\hline G353.381-00.114 & $\mathrm{Y}$ & $\cdots$ & $\cdots$ & $\cdots$ & $\cdots$ & $\mathrm{N}$ & G004.346+00.115 & $\mathrm{N}$ & $\mathrm{N}$ & $\cdots$ & $\cdots$ & $\cdots$ & $\mathrm{N}$ \\
\hline G353.398-00.391 & $\mathrm{N}$ & $\ldots$ & $\cdots$ & $\cdots$ & $\ldots$ & $\mathrm{N}$ & G004.412+00.118 & $\mathrm{Y}$ & $\mathrm{N}$ & $\ldots$ & $\cdots$ & $\cdots$ & $\mathrm{Y}$ \\
\hline G353.557-00.014 & $\mathrm{Y}$ & $\cdots$ & $\cdots$ & $\cdots$ & $\cdots$ & $\mathrm{Y}$ & G004.527-00.136 & $\mathrm{Y}$ & $\mathrm{N}$ & $\cdots$ & $\cdots$ & $\cdots$ & $\mathrm{Y}$ \\
\hline G354.200-00.054 & $\mathrm{Y}$ & $\cdots$ & $\cdots$ & $\cdots$ & $\cdots$ & $\mathrm{N}$ & G004.568-00.118 & $\mathrm{Y}$ & $\mathrm{N}$ & $\cdots$ & $\cdots$ & $\cdots$ & $\mathrm{N}$ \\
\hline $\mathrm{G} 354.418+00.036$ & $\mathrm{~N}$ & $\ldots$ & $\cdots$ & $\ldots$ & $\ldots$ & $\mathrm{N}$ & G005.193-00.284 & $\mathrm{Y}$ & Y & $\ldots$ & $\cdots$ & $\cdots$ & $\mathrm{N}$ \\
\hline $\mathrm{G} 354.486+00.085$ & $\mathrm{Y}$ & $\ldots$ & $\ldots$ & $\ldots$ & $\ldots$ & $\mathrm{Y}$ & G005.479-00.241 & $\mathrm{Y}$ & $\mathrm{N}$ & $\ldots$ & $\cdots$ & $\ldots$ & $\mathrm{Y}$ \\
\hline G354.588+00.007 & $\mathrm{Y}$ & $\ldots$ & $\ldots$ & $\ldots$ & $\mathrm{N}$ & $\mathrm{N}$ & G005.524 + 00.033 & $\mathrm{Y}$ & $\mathrm{Y}$ & $\ldots$ & $\ldots$ & $\ldots$ & $\mathrm{Y}$ \\
\hline $\mathrm{G} 354.610+00.484$ & $\mathrm{Y}$ & $\ldots$ & $\ldots$ & $\ldots$ & $\mathrm{N}$ & $\mathrm{Y}$ & G005.633+00.240 & $\mathrm{N}$ & $\mathrm{Y}$ & $\ldots$ & $\cdots$ & $\ldots$ & $\mathrm{N}$ \\
\hline G354.664 + 00.470 & $\mathrm{~N}$ & $\cdots$ & $\cdots$ & $\cdots$ & $\mathrm{N}$ & $\mathrm{N}$ & G005.899-00.427 & $\mathrm{N}$ & $\mathrm{Y}$ & $\cdots$ & $\cdots$ & $\cdots$ & $\mathrm{N}$ \\
\hline G354.665 + 00.247 & $\mathrm{N}$ & $\cdots$ & $\cdots$ & $\cdots$ & $\mathrm{Y}$ & $\mathrm{N}$ & G006.014-00.364 & $\mathrm{Y}$ & $\mathrm{N}$ & $\cdots$ & $\cdots$ & $\cdots$ & $\mathrm{N}$ \\
\hline G354.717+00.293 & $\mathrm{N}$ & $\cdots$ & $\ldots$ & $\ldots$ & $\mathrm{Y}$ & $\mathrm{N}$ & G006.083-00.117 & $\mathrm{Y}$ & $\mathrm{N}$ & $\ldots$ & $\ldots$ & $\ldots$ & $\mathrm{Y}$ \\
\hline G354.934+00.327 & $\mathrm{Y}$ & $\ldots$ & $\ldots$ & $\ldots$ & $\mathrm{Y}$ & $\mathrm{Y}$ & G006.148-00.635 & $\mathrm{Y}$ & $\mathrm{N}$ & $\ldots$ & $\ldots$ & $\ldots$ & $\mathrm{N}$ \\
\hline G354.979-00.528 & $\mathrm{N}$ & $\cdots$ & $\cdots$ & $\cdots$ & $\mathrm{N}$ & $\mathrm{N}$ & G006.160-00.608 & $\mathrm{Y}$ & $\mathrm{N}$ & $\cdots$ & $\cdots$ & $\cdots$ & $\mathrm{N}$ \\
\hline G355.242+00.096 & $\mathrm{Y}$ & $\cdots$ & $\cdots$ & $\mathrm{Y}$ & $\cdots$ & $\mathrm{Y}$ & G006.225-00.569 & $\mathrm{N}$ & $\mathrm{Y}$ & $\cdots$ & $\cdots$ & $\cdots$ & $\mathrm{N}$ \\
\hline G355.344+00.145 & $\mathrm{Y}$ & $\cdots$ & $\cdots$ & $\mathrm{Y}$ & $\cdots$ & $\mathrm{Y}$ & G006.398-00.474 & $\mathrm{N}$ & $\mathrm{Y}$ & $\cdots$ & $\cdots$ & $\cdots$ & $\mathrm{N}$ \\
\hline G355.532-00.100 & $\mathrm{Y}$ & $\cdots$ & $\cdots$ & $\mathrm{Y}$ & $\cdots$ & $\mathrm{N}$ & G006.553-00.095 & $\mathrm{Y}$ & Y & $\cdots$ & $\cdots$ & $\cdots$ & $\mathrm{Y}$ \\
\hline $\mathrm{G} 355.581+00.288$ & $\mathrm{Y}$ & $\ldots$ & $\cdots$ & $\mathrm{Y}$ & $\ldots$ & $\mathrm{Y}$ & G006.565-00.297 & $\mathrm{N}$ & $\mathrm{Y}$ & $\ldots$ & $\cdots$ & $\cdots$ & $\mathrm{Y}$ \\
\hline $\mathrm{G} 355.661+00.382$ & $\mathrm{Y}$ & $\ldots$ & $\ldots$ & $\mathrm{Y}$ & $\ldots$ & $\mathrm{N}$ & $\mathrm{G} 007.041+00.176$ & $\mathrm{Y}$ & $\mathrm{N}$ & $\ldots$ & $\ldots$ & $\ldots$ & $\mathrm{Y}$ \\
\hline G355.696+00.350 & $\mathrm{Y}$ & $\ldots$ & $\ldots$ & $\mathrm{Y}$ & $\ldots$ & $\mathrm{N}$ & $\mathrm{G} 007.176+00.087$ & $\mathrm{Y}$ & $\mathrm{N}$ & $\ldots$ & $\ldots$ & $\ldots$ & $\mathrm{N}$ \\
\hline G355.700-00.100 & $\mathrm{Y}$ & $\ldots$ & $\ldots$ & $\mathrm{N}$ & $\ldots$ & $\mathrm{N}$ & G007.254-00.073 & $\mathrm{Y}$ & $\mathrm{N}$ & $\cdots$ & $\cdots$ & $\cdots$ & $\mathrm{Y}$ \\
\hline $\mathrm{G} 355.734+00.138$ & $\mathrm{Y}$ & $\ldots$ & $\ldots$ & $\mathrm{Y}$ & $\ldots$ & $\mathrm{N}$ & $\mathrm{G} 007.266+00.183$ & $\mathrm{Y}$ & $\mathrm{Y}$ & $\ldots$ & $\cdots$ & $\ldots$ & $\mathrm{N}$ \\
\hline G355.801-00.253 & $\mathrm{Y}$ & $\cdots$ & $\cdots$ & $\mathrm{N}$ & $\cdots$ & $\mathrm{N}$ & G007.299-00.116 & $\mathrm{N}$ & $\mathrm{N}$ & $\cdots$ & $\cdots$ & $\cdots$ & $\mathrm{N}$ \\
\hline $\mathrm{G} 356.230+00.066$ & $\mathrm{Y}$ & $\ldots$ & $\ldots$ & $\mathrm{N}$ & $\ldots$ & $\mathrm{N}$ & G007.420+00.366 & $\mathrm{Y}$ & $\mathrm{N}$ & $\cdots$ & $\cdots$ & $\ldots$ & $\mathrm{Y}$ \\
\hline $\mathrm{G} 356.235+00.642$ & $\mathrm{Y}$ & $\cdots$ & $\cdots$ & $\mathrm{Y}$ & $\cdots$ & $\mathrm{N}$ & G007.466-00.279 & $\mathrm{Y}$ & $\mathrm{N}$ & $\cdots$ & $\cdots$ & $\cdots$ & $\mathrm{N}$ \\
\hline G356.307-00.210 & $\mathrm{Y}$ & $\cdots$ & $\cdots$ & $\mathrm{N}$ & $\cdots$ & $\mathrm{N}$ & $\mathrm{G} 007.472+00.060$ & $\mathrm{Y}$ & $\mathrm{N}$ & $\cdots$ & $\cdots$ & $\cdots$ & $\mathrm{Y}$ \\
\hline G356.470-00.001 & $\mathrm{Y}$ & $\cdots$ & $\cdots$ & $\mathrm{N}$ & $\cdots$ & $\mathrm{Y}$ & G007.700-00.079 & $\mathrm{N}$ & $\mathrm{N}$ & $\cdots$ & $\cdots$ & $\cdots$ & $\mathrm{N}$ \\
\hline G356.560-00.086 & $\mathrm{Y}$ & $\cdots$ & $\cdots$ & $\mathrm{N}$ & $\cdots$ & $\mathrm{N}$ & $\mathrm{G} 007.768+00.014$ & $\mathrm{Y}$ & $\mathrm{Y}$ & $\cdots$ & $\cdots$ & $\cdots$ & $\mathrm{N}$ \\
\hline G356.650+00.129 & $\mathrm{Y}$ & $\cdots$ & $\cdots$ & $\mathrm{Y}$ & $\cdots$ & $\mathrm{N}$ & G007.806-00.621 & $\mathrm{Y}$ & Y & $\cdots$ & $\cdots$ & $\cdots$ & $\mathrm{N}$ \\
\hline G357.484-00.036 & $\mathrm{N}$ & $\ldots$ & $\cdots$ & $\mathrm{N}$ & $\ldots$ & $\mathrm{Y}$ & G008.005-00.484 & $\mathrm{Y}$ & Y & $\ldots$ & $\cdots$ & $\cdots$ & $\mathrm{N}$ \\
\hline G357.970-00.169 & $\mathrm{Y}$ & $\ldots$ & $\ldots$ & $\mathrm{N}$ & $\ldots$ & $\mathrm{N}$ & G008.006-00.156 & $\mathrm{Y}$ & $\mathrm{Y}$ & $\ldots$ & $\ldots$ & $\ldots$ & $\mathrm{Y}$ \\
\hline G357.998-00.159 & $\mathrm{Y}$ & $\ldots$ & $\ldots$ & $\mathrm{N}$ & $\ldots$ & $\mathrm{N}$ & G008.094 + 00.085 & $\mathrm{~N}$ & $\mathrm{~N}$ & $\ldots$ & $\ldots$ & $\ldots$ & $\mathrm{N}$ \\
\hline G358.319-00.414 & $\mathrm{N}$ & $\cdots$ & $\cdots$ & $\mathrm{N}$ & $\cdots$ & $\mathrm{N}$ & $\mathrm{G} 008.103+00.340$ & $\mathrm{Y}$ & $\mathrm{N}$ & $\cdots$ & $\cdots$ & $\cdots$ & $\mathrm{N}$ \\
\hline G358.379-00.840 & $\mathrm{N}$ & $\cdots$ & $\ldots$ & $\mathrm{N}$ & $\cdots$ & $\mathrm{N}$ & $\mathrm{G} 008.137+00.228$ & $\mathrm{~N}$ & $\mathrm{~N}$ & $\cdots$ & $\cdots$ & $\cdots$ & $\mathrm{N}$ \\
\hline $\mathrm{G} 358.530+00.056$ & $\mathrm{~N}$ & $\cdots$ & $\mathrm{N}$ & $\mathrm{N}$ & $\cdots$ & $\mathrm{N}$ & G008.362-00.303 & $\mathrm{Y}$ & $\mathrm{N}$ & $\cdots$ & $\cdots$ & $\cdots$ & $\mathrm{N}$ \\
\hline G358.552-00.025 & $\mathrm{N}$ & $\cdots$ & $\mathrm{N}$ & $\mathrm{N}$ & $\cdots$ & $\mathrm{N}$ & G008.373-00.352 & $\mathrm{Y}$ & $\mathrm{Y}$ & $\cdots$ & $\cdots$ & $\ldots$ & $\mathrm{N}$ \\
\hline G358.616-00.076 & $\mathrm{Y}$ & $\cdots$ & $\mathrm{N}$ & $\mathrm{N}$ & $\ldots$ & $\mathrm{N}$ & G008.432-00.276 & $\mathrm{Y}$ & $\mathrm{N}$ & $\cdots$ & $\cdots$ & $\cdots$ & $\mathrm{Y}$ \\
\hline G358.623-00.066 & $\mathrm{Y}$ & $\cdots$ & $\mathrm{Y}$ & $\mathrm{N}$ & $\cdots$ & $\mathrm{N}$ & G008.666-00.351 & $\mathrm{Y}$ & $\mathrm{N}$ & $\cdots$ & $\cdots$ & $\cdots$ & $\mathrm{N}$ \\
\hline G358.633 + 00.062 & $\mathrm{Y}$ & $\cdots$ & $\mathrm{Y}$ & $\mathrm{N}$ & $\cdots$ & $\mathrm{N}$ & G008.830-00.715 & $\mathrm{Y}$ & $\mathrm{N}$ & $\cdots$ & $\cdots$ & $\cdots$ & $\mathrm{N}$ \\
\hline
\end{tabular}


Table 2

(Continued)

\begin{tabular}{|c|c|c|c|c|c|c|c|c|c|c|c|c|c|}
\hline Region & N3 & $\mathrm{CA}$ & $\mathrm{TD}$ & $+\mathrm{E} 135$ & $\mathrm{BC} 1$ & $\mathrm{~F} 3$ & Region & N3 & $\mathrm{CA}$ & TD & E135 & $\mathrm{BC} 1$ & F3 \\
\hline G358.652-00.078 & $\mathrm{N}$ & $\cdots$ & $\mathrm{N}$ & $\mathrm{N}$ & $\cdots$ & $\mathrm{N}$ & G008.865-00.323 & $\mathrm{Y}$ & $\mathrm{N}$ & $\cdots$ & $\cdots$ & $\cdots$ & $\mathrm{N}$ \\
\hline G358.680-00.087 & $\mathrm{N}$ & $\ldots$ & $\mathrm{Y}$ & $\mathrm{N}$ & $\ldots$ & $\mathrm{N}$ & G009.178+00.043 & $\mathrm{Y}$ & $\mathrm{N}$ & $\ldots$ & $\ldots$ & $\ldots$ & $\mathrm{N}$ \\
\hline G358.694-00.075 & $\mathrm{N}$ & $\cdots$ & $\mathrm{Y}$ & $\mathrm{N}$ & $\cdots$ & $\mathrm{N}$ & G009.615 + 00.198 & $\mathrm{Y}$ & $\mathrm{N}$ & $\cdots$ & $\cdots$ & $\cdots$ & $\mathrm{N}$ \\
\hline G358.720+00.011 & $\mathrm{Y}$ & $\cdots$ & $\mathrm{N}$ & $\mathrm{N}$ & $\cdots$ & $\mathrm{N}$ & G009.682+00.206 & $\mathrm{N}$ & $\mathrm{N}$ & $\cdots$ & $\cdots$ & $\cdots$ & $\mathrm{N}$ \\
\hline $\mathrm{G} 358.797+00.058$ & $\mathrm{Y}$ & $\ldots$ & $\mathrm{N}$ & $\mathrm{N}$ & $\ldots$ & $\mathrm{N}$ & G009.717-00.832 & $\mathrm{Y}$ & $\mathrm{N}$ & $\ldots$ & $\ldots$ & $\ldots$ & $\mathrm{N}$ \\
\hline $\mathrm{G} 358.827+00.085$ & $\mathrm{~N}$ & $\cdots$ & $\mathrm{N}$ & $\mathrm{N}$ & $\cdots$ & $\mathrm{N}$ & G009.741 + 00.842 & $\mathrm{Y}$ & $\mathrm{N}$ & $\cdots$ & $\cdots$ & $\cdots$ & $\mathrm{N}$ \\
\hline G359.159-00.038 & $\mathrm{Y}$ & $\cdots$ & $\mathrm{N}$ & $\mathrm{N}$ & $\cdots$ & $\mathrm{N}$ & G009.875-00.749 & $\mathrm{Y}$ & $\mathrm{Y}$ & $\cdots$ & $\cdots$ & $\cdots$ & $\mathrm{N}$ \\
\hline G359.277-00.264 & $\mathrm{N}$ & $\cdots$ & $\mathrm{N}$ & $\mathrm{N}$ & $\cdots$ & $\mathrm{N}$ & G009.925-00.745 & $\mathrm{Y}$ & $\mathrm{N}$ & $\cdots$ & $\cdots$ & $\cdots$ & $\mathrm{N}$ \\
\hline G359.432-00.086 & $\mathrm{Y}$ & $\cdots$ & $\mathrm{N}$ & $\mathrm{N}$ & $\cdots$ & $\mathrm{N}$ & G009.982-00.752 & $\mathrm{Y}$ & $\mathrm{N}$ & $\ldots$ & $\cdots$ & $\cdots$ & $\mathrm{N}$ \\
\hline G359.467-00.172 & $\mathrm{Y}$ & $\cdots$ & $\mathrm{N}$ & $\mathrm{N}$ & $\cdots$ & $\mathrm{Y}$ & $\cdots$ & $\cdots$ & $\cdots$ & $\cdots$ & $\cdots$ & $\cdots$ & $\cdots$ \\
\hline
\end{tabular}

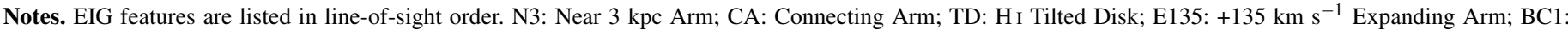
Bania's Clump 1; F3: Far 3 kpc Arm.

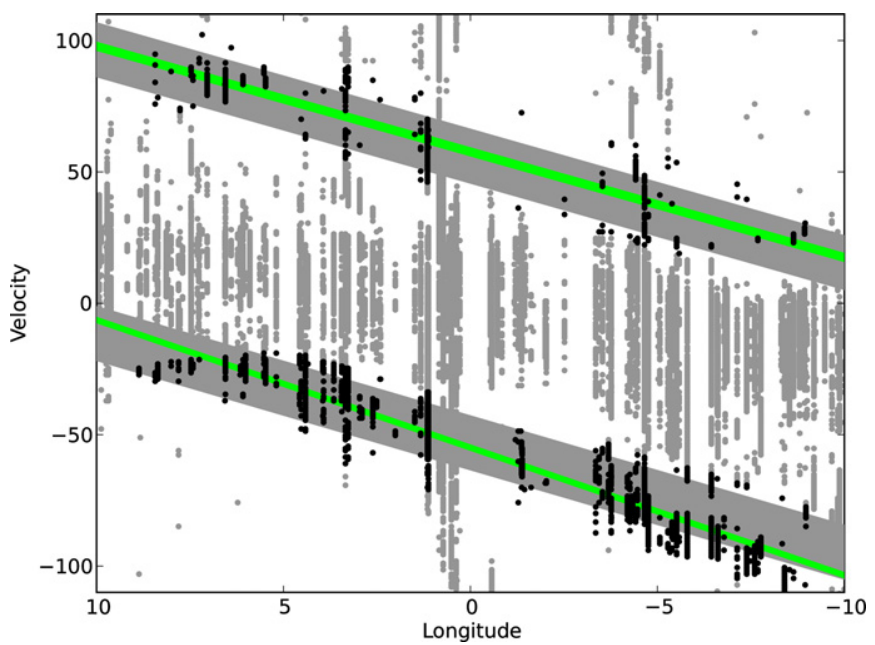

Figure 4. Velocity channels of significant $\mathrm{H}_{\mathrm{I}}$ absorption: those included in the tracing of the $3 \mathrm{kpc}$ Arms are shown in black, while those channels which were excluded from the analysis are in gray. The fits of Dame \& Thaddeus (2008) are given by the gray bands (see Figure 2), and the fits from this analysis are displayed in green.

(A color version of this figure is available in the online journal.)

spectrum of G007.176+00.087 (see Figure 1). The Near $3 \mathrm{kpc}$ Arm also displays evidence of bifurcation, in both the $\mathrm{HI}$ emission and absorption, near $l=358^{\circ}$ (see, for example, G358.616-00.076, G358.623-00.066, G358.633+00.062, and G359.432-00.086 in Figure 1).

\subsubsection{The Longitude-Velocity Location of the $3 \mathrm{kpc}$ Arms}

The locus of each of the $3 \mathrm{kpc}$ Arms, as traced by $\mathrm{HI}$ absorption, in $l v$ space was also investigated. Dame $\&$ Thaddeus (2008) provide $l v$ fits to the Near and Far $3 \mathrm{kpc}$ Arms and report a velocity dispersion of $21 \mathrm{~km} \mathrm{~s}^{-1}$ for both arms. However, they excluded large regions of longitude, within $|l|<10^{\circ}$, from the computation of the physical properties of each arm.

In order to investigate the locus of each arm in $l v$ space, a subset of absorption channels were selected for analysis (see Figure 4). We included all channels within $1^{\circ}<|l|<9^{\circ}$ (between $9^{\circ}<|l|<10^{\circ}$ there is ambiguity between the $3 \mathrm{kpc}$ Arms and the circular rotation velocities and for $|l|<1^{\circ}$ there is ambiguity with the Tilted Disk), which had velocities outside the envelope of allowed circular rotation velocities (i.e., $V_{\text {circular-rotation }}<|V|<110 \mathrm{~km} \mathrm{~s}^{-1}$ ) and were not associated

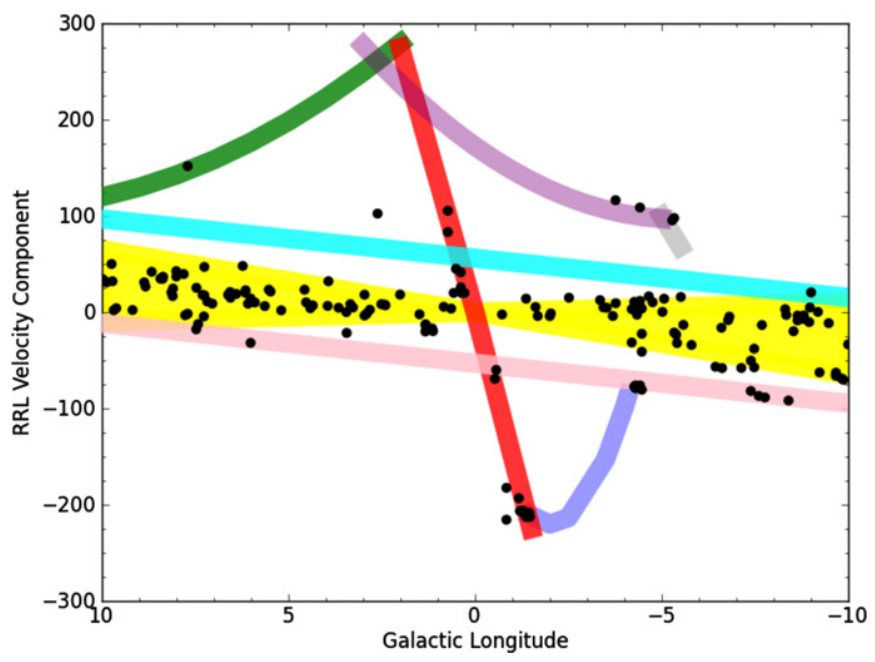

Figure 5. Longitude-velocity "crayon" diagram (see Figure 2) showing the distribution of $\mathrm{H}$ II region RRL velocity components.

(A color version of this figure is available in the online journal.)

with either of Bania's Clumps. Linear fits to these appear as Equations (3) and (4) for the Near and Far Arms, respectively.

Near 3 kpc Arm:

$$
\begin{gathered}
V_{\mathrm{N} 3 \mathrm{kpc}}=-59.2+4.12 l \pm 8.67 \\
V_{\mathrm{F} 3 \mathrm{kpc}}=+57.7+4.02 l \pm 15.61
\end{gathered}
$$

In both cases, the linear fits $( \pm 5 \sigma)$ of the structure as given by H I absorption are consistent with the Dame \& Thaddeus (2008) fits from $\mathrm{CO}$ emission (see Figure 4).

\section{LONGITUDE-VELOCITY DISTRIBUTION OF H II REGIONS TOWARD THE EXTREME INNER GALAXY}

$\mathrm{H}$ II regions provide radio continuum sources to measure $\mathrm{H}$ I absorption toward, but they also provide a secondary tracer of the EIG region - their own systemic velocities.

The $l v$ distribution of known $\mathrm{H}$ II regions has previously been investigated by Anderson et al. (2012), however, all $\mathrm{H}$ II regions with highly non-circular motions (i.e., those of interest to this work) were excluded from their analysis. The $l v$ distribution of $\mathrm{HII}_{\text {II }}$ region RRL velocity components used in this work is shown in Figure 5-note that for $\mathrm{H}$ II regions with multiple velocity components, all are shown. 
Table 3

H II Regions with an RRL Velocity Associated (in $l v$ Space) with Known EIG Structure(s)—see Figure 5

\begin{tabular}{|c|c|c|c|c|}
\hline H II Region & & $V_{\mathrm{RRL}}$ & Reference & Association \\
\hline \multicolumn{5}{|c|}{ Single RRL velocity component $\mathrm{H}$ II regions } \\
\hline G350.996-00.557 & & +20.5 & 2 & Far 3 kpc Arm \\
\hline G351.601-00.348 & & -91.8 & 3 & Near 3 kpc Arm \\
\hline G352.233-00.151 & & -88.6 & 1 & Near 3 kpc Arm \\
\hline G352.398-00.057 & & -87.0 & 2 & Near 3 kpc Arm \\
\hline G352.611-00.172 & & -81.9 & 2 & Near 3 kpc Arm \\
\hline G354.665+00.247 & & +97.8 & 2 & Bania's Clump 1? \\
\hline G354.717+00.293 & & +95.3 & 1 & Bania’s Clump 1? \\
\hline G355.700-00.100 & & -76.1 & 2 & Near 3 kpc Arm \\
\hline $\mathrm{G} 356.235+00.642$ & & +116.3 & 2 & $+135 \mathrm{~km} \mathrm{~s}^{-1}$ Arm \\
\hline G358.530+00.056 & & -212.6 & 1 & Looping Ridge \\
\hline G358.552-00.025 & & -208.5 & 1 & Looping Ridge \\
\hline G358.616-00.076 & & -212.6 & 1 & Looping Ridge \\
\hline G358.623-00.066 & & -212.0 & 3 & Looping Ridge \\
\hline G358.652-00.078 & & -211.2 & 1 & Looping Ridge \\
\hline G358.680-00.087 & & -208.3 & 1 & Looping Ridge \\
\hline G358.694-00.075 & & -207.8 & 1 & Looping Ridge \\
\hline G358.720+00.011 & & -206.1 & 1 & Looping Ridge \\
\hline G358.797+00.058 & & -206.6 & 2 & Looping Ridge \\
\hline G358.827+00.085 & & -193.3 & 1 & Looping Ridge \\
\hline G359.432-00.086 & & -60.0 & 3 & Near 3 kpc Arm \\
\hline G359.467-00.172 & & -69.3 & 1 & Blended EIG features \\
\hline G000.510-00.051 & & +45.0 & 4 & Far 3 kpc Arm \\
\hline G003.439-00.349 & & -21.6 & 1 & Near 3 kpc Arm? \\
\hline G007.472+00.060 & & -17.8 & 2 & Near 3 kpc Arm \\
\hline \multicolumn{5}{|c|}{ Multiple RRL velocity component $\mathrm{H}$ II regions } \\
\hline G355.532-00.100 & $\mathbf{a}$ & +3.8 & 1 & $\cdots$ \\
\hline G355.532-00.100 & $\mathrm{b}$ & -22.5 & 1 & $\cdots$ \\
\hline G355.532-00.100 & $\mathrm{c}$ & -82.6 & 1 & Near 3 kpc Arm \\
\hline G355.532-00.100 & $\mathrm{d}$ & -41.1 & 1 & $\cdots$ \\
\hline $\mathrm{G} 355.581+00.028$ & $\mathrm{a}$ & 108.7 & 1 & $+135 \mathrm{~km} \mathrm{~s}^{-1}$ Arm \\
\hline G355.581+00.028 & $\mathrm{b}$ & -76.1 & 1 & Near 3 kpc Arm \\
\hline G355.581+00.028 & $\mathrm{c}$ & 11.7 & 1 & $\ldots$ \\
\hline G355.696+00.350 & $\mathrm{a}$ & +3.0 & 1 & $\cdots$ \\
\hline G355.696+00.350 & $\mathrm{b}$ & -79.1 & 1 & Near 3 kpc Arm \\
\hline G355.734+00.138 & $\mathrm{a}$ & +10.7 & 1 & $\cdots$ \\
\hline G355.734+00.138 & $\mathrm{b}$ & -77.4 & 1 & Near 3 kpc Arm \\
\hline G359.159-00.038 & $\mathrm{a}$ & -182.5 & 1 & Blended EIG features \\
\hline G359.159-00.038 & $\mathrm{b}$ & -215.6 & 1 & Blended EIG features \\
\hline G000.729-00.123 & $\mathrm{a}$ & +105.3 & 1 & Tilted Disk \\
\hline G000.729-00.123 & $\mathrm{b}$ & +83.2 & 1 & $\cdots$ \\
\hline G006.014-00.364 & $\mathrm{a}$ & +14.2 & 1 & $\cdots$ \\
\hline G006.014-00.364 & $\mathrm{b}$ & -31.9 & 1 & Near 3 kpc Arm \\
\hline G007.700-00.079 & $\mathrm{a}$ & -1.7 & 5 & $\ldots$ \\
\hline G007.700-00.079 & $\mathrm{b}$ & +151.7 & 5 & Connecting Arm \\
\hline
\end{tabular}

References for $V_{\text {RRL }}$ are as follows: (1) GBTHRDS 2011; (2) Lockman 1989; (3) Caswell \& Haynes 1987; (4) Downes et al. 1980; (5) Lockman et al. 1996.

Just as the distribution of $\mathrm{H}$ I absorption was closely associated with known $l v$ features in the EIG region (Section 5 and Figure 3 ), the systemic velocities of $\mathrm{H}$ II regions also trace these structures.

The circular rotation allowed velocities (yellow envelope in "crayon plots") account for $\sim 85 \%$ of the H II region RRL velocity components. Green et al. (2011), in a study of $6.7 \mathrm{GHz}$ methanol masers near the EIG, find the same velocity range accounts for $\sim 79 \%$ of their sample. However, only $\sim 10 \%$ of $\mathrm{H}$ II regions with a single RRL velocity component are associated with EIG features. A list of $\mathrm{H}$ II regions with RRL velocities associated with an EIG structure appears in Table 3.

\subsection{H II Regions Associated with $R_{\mathrm{Gal}} \lesssim 4$}

Until recently, it was believed that there are no known $\mathrm{H}$ II regions inside of the $3 \mathrm{kpc}$ Arms, except in the Tilted Disk (Rodríguez-Fernández 2006). Green et al. (2011) found no significant $6.7 \mathrm{GHz}$ methanol maser emission toward the $+135 \mathrm{~km} \mathrm{~s}^{-1}$ Expanding Arm or the Connecting Arm, suggesting that the features are primarily gas that is not undergoing high-mass star formation. This is in keeping with observations of other early-type barred galaxies which show star formation in the central nuclear region and at the ends of the bar, but not in the dust lanes along the bar (Rodriguez-Fernandez et al. 2006).

Using the collated $\mathrm{H}$ II region catalog of Paladini et al. (2003), Rodriguez-Fernandez et al. (2006) found no H II regions associated with structures outside the Nuclear Disk within $|l|<2^{\circ}$. The GBTHRDS recorded RRL velocity components from 21 previously unknown $H$ II regions within $|l|<2^{\circ}$, many (especially in Quadrant IV) with non-circular velocities-these $\mathrm{H}$ II regions are included in the target list of this work. However, as the Rodriguez-Fernandez et al. (2006) study found, these new $\mathrm{H}$ II regions are associated (in $l v$ space) with the Nuclear Disk and Looping Ridge intersection. Rodriguez-Fernandez et al. (2006) then investigated a wider longitude range, but could not identify any dust lane associated $\mathrm{H}$ II regions. It should be noted that Rodriguez-Fernandez et al. (2006) did not rule out the possibility of undetected ultra-compact $\mathrm{H}$ II regions in the dust lanes.

One diffuse HiI region, G007.700-0.079, identified by Lockman et al. (1996; but not included in the Paladini et al. 2003 catalog used in the Rodriguez-Fernandez et al. 2006 study) appears to have one of its RRL velocity components associated with the Connecting Arm. There is also evidence of two $6.7 \mathrm{GHz}$ methanol masers, tracers of current high-mass star formation, in the same part of $l v$ space (see Figure 1 of Green et al. 2011). In addition, there are four $\mathrm{H}$ II region RRL velocity components associated with the $+135 \mathrm{~km} \mathrm{~s}^{-1}$ Expanding Arm and/or Bania's Clump 1 (at $l, v=\sim-4^{\circ}, \sim 100 \mathrm{~km} \mathrm{~s}^{-1}$ ), as well as two $6.7 \mathrm{GHz}$ methanol masers from the Methanol Multibeam survey (cited in Green et al. 2011), however, only two of these regions have single RRL velocity components (multi-RRL component H II regions are probably the result of blending multiple emission sources along the line of sight). Therefore, there is evidence of some recent star formation in these structures.

The other H II region of note is G002.611+0.135 as it is the only HiI region that distinctly lies outside the "crayon" lines that delineate EIG structures in Figure 3. RodriguezFernandez et al. (2006) suggest that G002.611+0.135 could be associated with either their structure "J" or Bania's Clump 2 (see Figure 4 of Rodriguez-Fernandez et al. 2006). The latitude of the $\mathrm{H}$ II region suggests a stronger association with Clump 2.

\subsection{H II Regions Associated with the $3 \mathrm{kpc}$ Arms}

Only recently has there been evidence of significant star formation (Green et al. 2009) and large numbers of H II regions (Bania et al. 2010) in the $3 \mathrm{kpc}$ Arms. In emission from molecular clouds the signatures of the $3 \mathrm{kpc}$ Arms are clearly seen (Bania 1980), but the GBTHRDS was unable to discover many new H II regions, in either of the arms. However, both arms demonstrate high-mass star formation as traced by about fifty 6.7 GHz methanol masers (Green et al. 2009).

The certainty of associating $\mathrm{H}_{\mathrm{II}}$ regions with the $3 \mathrm{kpc}$ Arms (in $l v$ space) is best in the longitude range of this study $\left(|l|<10^{\circ}\right)$, as outside this limit the expected velocities of the 
$3 \mathrm{kpc}$ Arms overlap with circular-motion spiral arm models and the association becomes more ambiguous (Green et al. 2009).

Inside $|l|<10^{\circ}$ there are $11 \mathrm{H}$ II region $\mathrm{RRL}$ velocity components consistent with the Near $3 \mathrm{kpc}$ Arm and 2 consistent with the Far $3 \mathrm{kpc}$ Arm. This small number of RRL components does not allow for a repetition of the analysis of Section 5.1.1 using $\mathrm{H}$ II region RRL components rather than $\mathrm{H}$ I absorption.

\section{DISTANCE CONSTRAINTS FOR H II REGIONS FROM H I ABSORPTION}

The analysis of an $\mathrm{HI}$ absorption spectrum toward an $\mathrm{H}$ II region can constrain the line-of-sight distance to the $\mathrm{H}$ II itself.

Due to the lack of a reliable rotation model for the inner $\sim 3 \mathrm{kpc}$ of the Milky Way, kinematic distances to objects near, or in, the EIG are the most difficult to ascertain. However, it should be possible to provide distance constraints for $\mathrm{H}$ II regions with allowed circular rotation systemic velocities, using $\mathrm{H}$ I absorption associated with EIG features as approximate distance indicators.

In the inner Galaxy, inside the solar circle, each velocity corresponds to two degenerate solutions for the kinematic distance-each equidistant from the tangent (subcentral) point. This kinematic distance ambiguity can be resolved in cases where $\mathrm{HI}$ absorption is present at the velocity of a known structure-which indicates the $\mathrm{H}$ II region must be located behind the absorbing gas.

The distance arrangement of EIG features, listed in Section 4.1, from the literature is assumed to be Near $3 \mathrm{kpc}$ Arm, Connecting Arm, Tilted Disk, Looping Ridge, $+135 \mathrm{~km} \mathrm{~s}^{-1}$ Expanding Arm (and Bania's Clump 1), and Far 3 kpc Arm (however, not all structures are expected along any single line of sight; see Figure 2).

Therefore, if $\mathrm{H}$ r absorption is seen at velocities corresponding to a particular feature, the $\mathrm{H}$ II region must lie in or beyond that structure. In this way, we use $\mathrm{H}$ I absorption as an indicator of the lower limit of the line-of-sight distance, $D_{\text {los }}$. The RRL velocity of an $\mathrm{H}$ II region also hints at its location and Table 3 lists those $\mathrm{H}$ II regions with systemic velocities beyond the range expected by circular rotation (see Figure 5) and associated with EIG feature(s).

A discussion of each $\mathrm{H}$ II region appears in the Appendix.

\subsection{Kinematic Distances of Selected H II Regions}

For H II regions with systemic velocities associated with normal circular disk rotation (i.e., in the yellow envelope in Figure $5, R_{\mathrm{Gal}}>4 \mathrm{kpc}$ ), a Kinematic Distance Ambiguity Resolution (KDAR) is attempted. If a KDAR is achieved, the kinematic distance to the $\mathrm{H}$ iI region can then be calculated.

KDARs were achieved following these rules.

1. If the HII region RRL is consistent with normal circular disk rotation, and not with any EIG feature (see Table 3; i.e., no kinematic distances are calculated for regions with $\left.R_{\mathrm{Gal}}<3 \mathrm{kpc}\right)$.

2. Far: if the Hi absorption spectrum displays sufficient absorption associated with EIG features, then the $\mathrm{H}$ II region must be located at the "far" kinematic location, i.e., beyond the tangent point along the line of sight.

(a) If $\left|V_{\mathrm{RRL}}\right|<10 \mathrm{~km} \mathrm{~s}^{-1}$ a KDAR is attempted only if the $\mathrm{H}$ I absorption spectrum displays absorption associated with the Far $3 \mathrm{kpc}$ Arm, this is imposed in order to avoid confusion with EIG features at small velocities. (b) Quality "A" far-side KDARs were awarded to H II regions with statistically significant absorption in EIG features including at least the Near and Far $3 \mathrm{kpc}$ Arms.

(c) Quality "B" far-side KDARs were awarded to H II regions with statistically significant absorption in any EIG feature located on the far side of the GC.

(d) Quality "C" far-side KDARs were awarded to H II regions with large uncertainties $(>50 \%)$ in their calculated $D_{\text {los }}$ value (see Section 7.1.1).

3. Near: if the H I absorption spectrum displays no absorption associated with any EIG features, then it must be located at the "near" kinematic location. Here we assume that all the EIG features are visible within the latitude range of the target $\mathrm{H}$ II regions $(|b|<1.5)$. Note that the linear scale heights of the Near and Far $3 \mathrm{kpc}$ Arms is 103 pc FWHM (Dame \& Thaddeus 2008); assuming that the Far $3 \mathrm{kpc}$ Arm is at a uniform line-of-sight distance of $11.5 \mathrm{kpc}$, this scale height corresponds to a latitude range $|b| \lesssim 0.5$. As a result, if an $\mathrm{H}$ I absorption spectrum toward an $\mathrm{H}$ II region located at $|b|>0.5$ displays no absorption associated with any EIG feature, it is awarded a Near KDAR of Quality " $C$."

(a) KDARs of Quality "A" were given to all near-side H II regions, unless

(b) the calculated $D_{\text {los }}$ value had large uncertainties (>50\%), then a Quality "C" KDAR was given.

4. No KDAR was attempted for $\mathrm{H}$ II regions with multiple RRL velocity components, as multiple systemic velocities suggest several ionization sources along the line of sight. Note: multi-RRL velocity component $\mathrm{H}$ II regions account for less than $10 \%$ of the $\mathrm{H}$ II regions within $|l|<10^{\circ}$, compared with $30 \%$ for the Galactic plane in general (GBTHRDS).

$\mathrm{H}$ II regions with calculated kinematic distances are listed in Table 4.

Four H II regions (G350.177+00.017, G350.330+00.157, G353.557-00.014, and G003.949-00.100) were deemed to lie at the far kinematic location, beyond the EIG, following the rules above. However, these four regions have $R_{\mathrm{Gal}}<3 \mathrm{kpc}$, i.e., they are outside the bounds of the McClure-Griffiths \& Dickey (2007) rotation model and are therefore not included in Table 4.

If an $\mathrm{H}$ II region is awarded a far-side KDAR, based on the above requirements, a kinematic distance can be calculated using a Galactic rotation curve model (which assumes circular rotation). The IAU Galactic constants have been applied in these calculations: $R_{0}=8.5 \mathrm{kpc}$ and $\Theta_{0}=220 \mathrm{~km} \mathrm{~s}^{-1}$.

If an $\mathrm{H}$ II region must be located at least as far as the subcentral point, then its location inside, or beyond, the solar circle is given by its systemic velocity. In the inner Galaxy, velocities are positive in the first quadrant and negative in the fourth. The signs are reversed in the outer Galaxy, such that first quadrant sources located beyond the solar circle will have negative velocities, and fourth quadrant sources in the outer Galaxy will have positive velocities.

Throughout this work, the rotation curve of McClure-Griffiths $\&$ Dickey (2007) is used for regions within the solar circle. In the outer Galaxy, $D_{\text {los }}$ was calculated using a flat rotation model $\Theta_{R_{\text {Gal }}}=\Theta_{0}$.

\subsubsection{Kinematic Distance Uncertainties}

We follow the distance uncertainty analysis method of Anderson et al. (2012), investigating the effects of the choice 
Table 4

H II Regions with Calculated Kinematic Distances (in kiloparsecs)

\begin{tabular}{|c|c|c|c|c|c|}
\hline H II Region & $V_{\mathrm{RRL}}$ & Reference & $Q$ & $R_{\mathrm{Gal}}$ & $D_{\text {los }}$ \\
\hline \multicolumn{6}{|c|}{ KDAR: far-within rotation model boundaries } \\
\hline G350.813-00.019 & +0.3 & 3 & B & 8.6 & $16.9 \pm 4.2$ \\
\hline G351.028+00.155 & +4.8 & 1 & A & 9.7 & $18.0 \pm 5.9$ \\
\hline G351.358+00.666 & -3.6 & 3 & $\mathrm{~B}$ & 8.2 & $16.5 \pm 3.6$ \\
\hline G352.313-00.440 & -13.4 & 1 & A & 5.9 & $14.3 \pm 2.6$ \\
\hline G354.486+00.085 & +15.8 & 3 & $\mathrm{C}$ & 8.8 & $23.3 \pm *$ \\
\hline G354.610+00.484 & -23.4 & 1 & A & 3.9 & $12.3 \pm 2.2$ \\
\hline G354.934+00.327 & +14 & 4 & $\mathrm{C}$ & 14.6 & $23.1 \pm *$ \\
\hline G355.242+00.096 & +10.3 & 3 & $\mathrm{C}$ & 13.3 & $21.7 \pm *$ \\
\hline G355.344+00.145 & +16.4 & 1 & $\mathrm{C}$ & 16.8 & $24.8 \pm *$ \\
\hline G000.640+00.623 & +3.7 & 2 & $\mathrm{C}$ & 3.3 & $11.8 \pm *$ \\
\hline G000.838+00.189 & +5.6 & 1 & $\mathrm{C}$ & 3.0 & $11.5 \pm *$ \\
\hline G003.270-00.101 & +4.9 & 3 & $\mathrm{C}$ & 6.3 & $14.7 \pm *$ \\
\hline G003.342-00.079 & +8.3 & 3 & A & 5.2 & $13.6 \pm 6.7$ \\
\hline G004.412+00.118 & +4.1 & 3 & $\mathrm{C}$ & 7.1 & $15.6 \pm 8.9$ \\
\hline G004.527-00.136 & +10.2 & 1 & A & 5.4 & $13.8 \pm 3.9$ \\
\hline G005.479-00.241 & +21.4 & 3 & A & 4.1 & $12.5 \pm 2.3$ \\
\hline G005.524+00.033 & +23.3 & 1 & A & 4.0 & $12.3 \pm 2.2$ \\
\hline G006.083-00.117 & +8.8 & 3 & A & 6.3 & $14.7 \pm 3.5$ \\
\hline G006.553-00.095 & +15 & 3 & A & 5.3 & $13.7 \pm 2.5$ \\
\hline G006.565-00.297 & +20.9 & 3 & $\mathrm{~B}$ & 4.6 & $12.9 \pm 2.2$ \\
\hline G007.041+00.176 & +8.9 & 1 & A & 6.6 & $14.9 \pm 3.1$ \\
\hline G007.420+00.366 & -12.3 & 1 & $\mathrm{C}$ & 12.2 & $20.6 \pm *$ \\
\hline G008.006-00.156 & +42.6 & 3 & A & 3.4 & $11.6 \pm 1.9$ \\
\hline G008.432-00.276 & +34.8 & 1 & A & 4.0 & $12.2 \pm 2.0$ \\
\hline \multicolumn{6}{|c|}{ KDAR: near } \\
\hline G351.192+00.708 & -3.4 & 3 & $\mathrm{C}$ & 8.2 & $0.3 \pm *$ \\
\hline G353.186+00.887 & -4.7 & 3 & $\mathrm{C}$ & 7.6 & $0.9 \pm *$ \\
\hline G359.277-00.264 & -2.4 & 5 & $\mathrm{C}$ & 4.5 & $4.0 \pm *$ \\
\hline G005.889-00.427 & +10.1 & 3 & $\mathrm{C}$ & 6.0 & $2.5 \pm *$ \\
\hline G008.137+00.228 & +20.6 & 3 & $\mathrm{C}$ & 5.1 & $3.5 \pm 2.2$ \\
\hline G008.666-00.351 & +49.1 & 3 & A & 4.7 & $3.9 \pm 1.4$ \\
\hline G008.830-00.715 & +26.6 & 1 & $\mathrm{C}$ & 4.7 & $3.9 \pm 2.0$ \\
\hline
\end{tabular}

Notes. Errors in kinematic distances marked with asterisks $(*)$ denote errors which are $>100 \%$; note that while these errors are large, the significance of the KDAR remains.

References for the RRL velocities are as follows: (1) GBTHRDS 2011; (2) Lockman et al. 1996; (3) Lockman 1989; (4) Caswell \& Haynes 1987; (5) Wink et al. 1982.

of rotation curve, streaming motions, and solar rotation speed on kinematic distance calculations. We compare all sources of uncertainty to the distances calculated from the rotation model of McClure-Griffiths \& Dickey (2007).

First, we compute, for a grid of $(l, v)$ positions, the kinematic distance using the rotation curves of McClure-Griffiths \& Dickey (2007), Brand \& Blitz (1993), and Clemens (1985). The standard deviation of these distances for each $(l, v)$ is then computed and divided by the McClure-Griffiths \& Dickey (2007) distance to obtain the percentage uncertainty due to choice of rotation curve. We assessed a grid of $|l|<10^{\circ}$ and $|v|<200 \mathrm{~km} \mathrm{~s}^{-1}$ with steps of 0.1 in each unit.

This procedure is then repeated, but instead of varying the Galactic rotation model, the percentage uncertainty due to streaming motions (of $15 \mathrm{~km} \mathrm{~s}^{-1}$ ) and an altered circular solar rotation speed of $255 \mathrm{~km} \mathrm{~s}^{-1}$ (Reid et al. 2009) are investigated.

The effect of these three sources of error are then added in quadrature for each $(l, v)$ pair, before transformation onto a face-on plot (Figure 6).

It should be noted that kinematic distance errors at small Galactic longitudes are intrinsically larger than for other sections

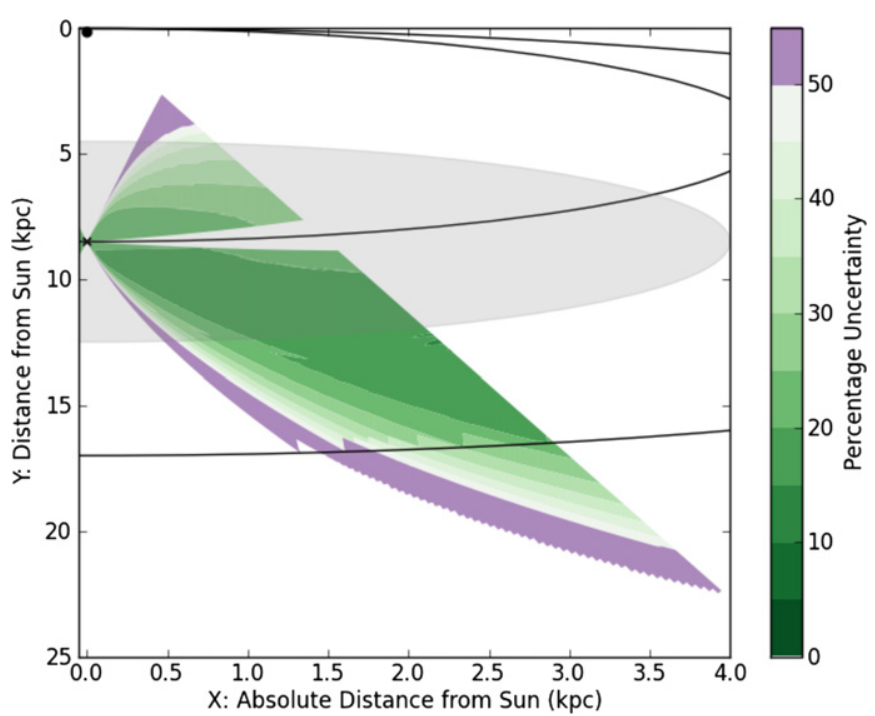

Figure 6. Total percentage uncertainty in the line-of-sight distance $D_{\text {los }}$ caused by the choice of rotation curve, non-circular streaming motions of $15 \mathrm{~km} \mathrm{~s}^{-1}$, and by changing the solar circular rotation speed to $\Theta_{o}=255 \mathrm{~km} \mathrm{~s}^{-1}$. Blank areas are indicative of $|l|>10^{\circ}$ (where no error analysis was carried out), or percentage uncertainties $>100 \%$. The EIG is shaded gray (no kinematic distances were calculated for this region), and the solar circle and locus of tangent points appear as the black circles. The percentage uncertainties are mirrored for lines of sight in the fourth quadrant, here, only $l>0$ is shown for clarity.

(A color version of this figure is available in the online journal.)

of the Galaxy. Equal steps in velocity map to unequal length steps in $D_{\text {los }}$, such that $d D_{\text {los }} / d v \propto \sin ^{-1} l$. This can be seen in Figure 6 where the percentage uncertainty is higher for smaller longitudes.

Uncertainties due to rotation curve. In addition to larger uncertainties at small longitudes due to the velocity gradient, errors are also larger in the outer Galaxy due to the uncertainty in the outer Galaxy circular rotation models. Flat, rising, and falling rotation curves have been suggested for beyond the solar circle (Brand \& Blitz 1993; Honma et al. 2007; Hachisuka et al. 2009). Here we have used the rotation models of McClure-Griffiths \& Dickey (2007), Brand \& Blitz (1993), and Clemens (1985). Note that the McClure-Griffiths \& Dickey (2007) model has an applicable Galactocentric range of $3 \leqslant R_{\mathrm{Gal}} \leqslant 8 \mathrm{kpc}$. As a result the model was extrapolated to the solar circle, and a flat rotation curve was assumed for $R_{\mathrm{Gal}}>R_{o}$. The largest discrepancy between these three models occurs at $R_{\mathrm{Gal}} \sim 10 \mathrm{kpc}$, but even at $R_{\mathrm{Gal}}<R_{o}$ the models differ significantly.

Uncertainties due to non-circular, streaming motions. Large non-circular motions have precluded Galactic astronomers from fitting a rotation curve to the EIG. Smaller-scale non-circular motions are ubiquitous in the Galaxy and are the result of systematic velocity fields within a source, or ordered largescale Galactic streaming motions (Anderson et al. 2012). Bania \& Lockman (1984) suggest an uncertainty, due to non-circular motions, of 5-10 $\mathrm{km} \mathrm{s}^{-1}$; whereas Kolpak et al. (2003) assign an estimate of cloud-cloud dispersion of $5 \mathrm{~km} \mathrm{~s}^{-1}$ in addition to contributions from Galactic scale streaming motions of $10 \mathrm{~km} \mathrm{~s}^{-1}$. Dickey et al. (2003) and Jones \& Dickey (2012) find $\mathrm{H}$ I absorption components extending to $10-20 \mathrm{~km} \mathrm{~s}^{-1}$ beyond the systemic velocity of $\mathrm{H}$ II regions.

In order to promote a conservative approach to kinematic distance uncertainties, the random uncertainty due to noncircular motions is set to $15 \mathrm{~km} \mathrm{~s}^{-1}$. The contribution of errors due to streaming motions in relation to the total uncertainty in 

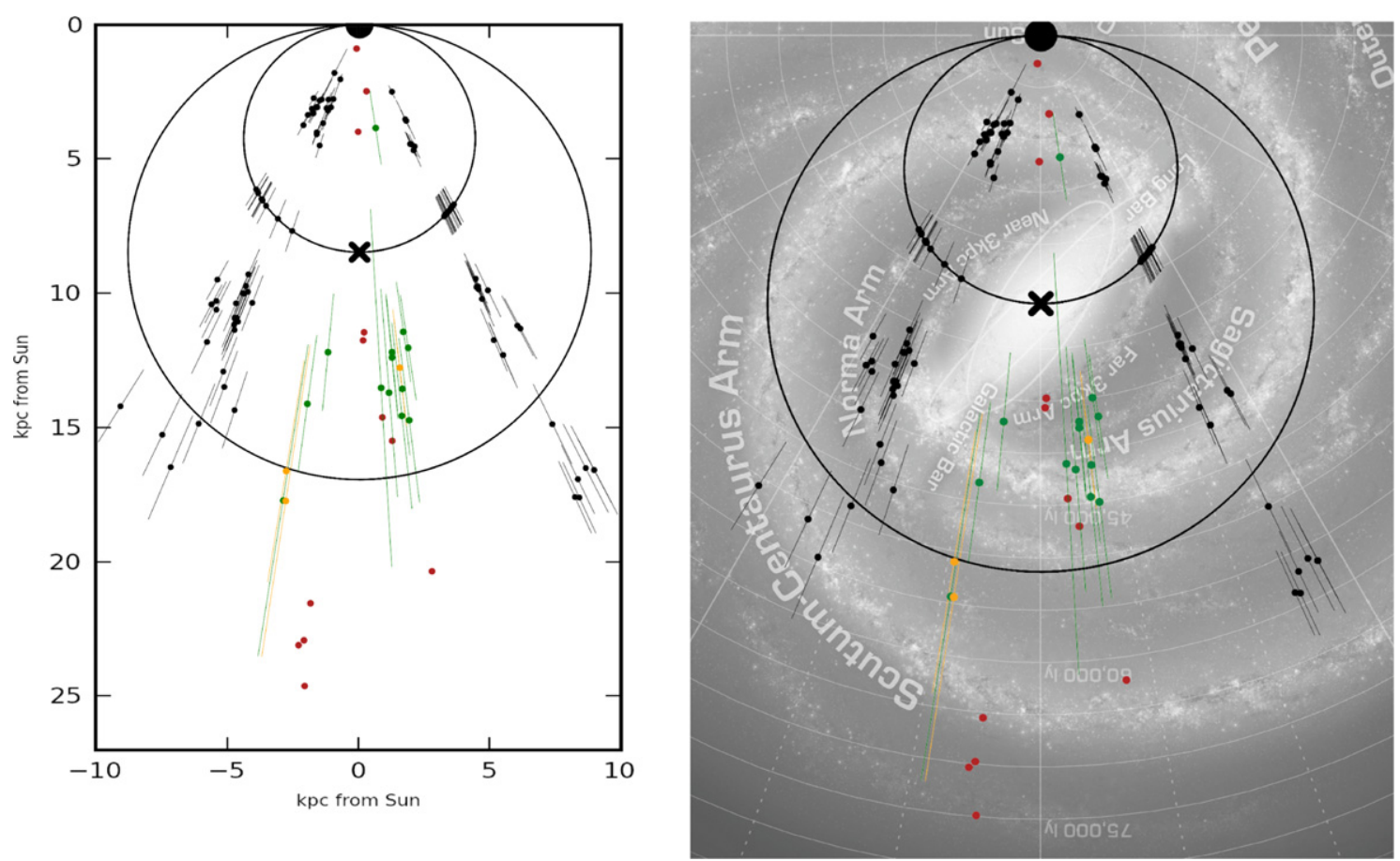

Figure 7. Positions of the $\mathrm{H}$ II region complexes for which a kinematic distance was calculated (quality A, B, and C shown as green, orange, and red markers, respectively). Also shown are the solar circle and locus of tangent points (black circles) and kinematic distances for H II regions from Jones \& Dickey (2012; black markers). Error bars are calculated according to the analysis of Section 7.1.1; the large uncertainties are not shown for quality C distances. Background image credit (right panel): Hurt \& Benjamin in Churchwell et al. (2009).

(A color version of this figure is available in the online journal.)

kinematic distance is high, especially for small longitudes. The errors due to non-circular motions are the standard deviation of the three $(l, v)$ grids, $(l, v),(l, v+15),(l, v-15)$ divided by the $(l, v)$ distance, all computed with the McClure-Griffiths \& Dickey (2007) rotation model.

Uncertainties due to solar rotation parameters. The IAU values for $R_{o}=8.5 \mathrm{kpc}$ and $\Theta_{o}=220 \mathrm{~km} \mathrm{~s}^{-1}$ have been used throughout this work. However, here we investigate the significance of an altered solar rotation speed, as suggested by Reid et al. (2009). Two $(l, v)$ grids were computed with the rotation model of McClure-Griffiths \& Dickey (2007), using $\Theta_{o}=220,250 \mathrm{~km} \mathrm{~s}^{-1}$. The standard deviation of these two grids, at each locus, was then divided by the standard (i.e., $\Theta_{o}=220 \mathrm{~km} \mathrm{~s}^{-1}$ ) distance to compute the percentage uncertainty due to choice of solar rotation parameters. Note that the Reid et al. (2009) value for $R_{o}=8.4 \pm 0.6 \mathrm{kpc}$ is consistent with the IAU value, and is therefore not investigated here.

\subsection{Galactic Distribution of H II Regions}

In order to examine the large-scale structure of the Galaxy, $\mathrm{H}$ II regions with successfully calculated kinematic distances were transformed into a face-on map of the Milky Way (left panel of Figure 7) and also superimposed onto an artist's conception of the Galaxy (right panel). The background image used in the right panel of Figure 7 was created using stellar, H I, and CO data (Churchwell et al. 2009) and was reviewed in Urquhart et al. (2012).

In addition, the kinematic distances from Jones \& Dickey (2012) are also displayed. Figure 7 demonstrates the need for $\mathrm{H}$ II region discovery and KDAR studies for Galactic longitudes $340^{\circ}<l<350^{\circ}$ in order to further investigate the end of the bar and differentiate the Norma and Near $3 \mathrm{kpc}$ Arms, as well as the Sagittarius and Perseus Arms on the far side of the locus of subcentral points (smaller circle in Figure 7). At the end of the bar in the first quadrant, $\mathrm{H}$ II region KDARs have been made by Anderson \& Bania (2009) and Bania et al. (2012) - further encouragement for a fourth quadrant study.

Recently, Dame \& Thaddeus (2011) identified an extension of the Scutum-Centaurus Arm at extreme distances from the Sun, in the first Galactic quadrant. However, confirmation of this discovery requires tracing the arm over its entire longitude range. Dame \& Thaddeus (2011) comment that molecular gas which constitutes the section of Scutum-Centaurus Arm behind the $\mathrm{GC}$ will be the most difficult to deconvolve. In the longitude range of this paper, $\mathrm{H}$ II regions with systemic velocities opposite in sign to circular-disk rotation must be located in the EIG or beyond the solar circle. Using $\mathrm{HI}$ absorption features to resolve this ambiguity has allowed for seven H II regions to be unequivocally placed in the outer Galaxy. Several of these outer Galaxy regions (see Figure 7) appear to trace the Scutum-Centaurus Arm.

\section{SUMMARY}

The EIG remains a difficult section of the Milky Way to study. In terms of Galactic structure, kinematic studies in this region are hampered by a lack of rotation model for $R_{\mathrm{Gal}}<4 \mathrm{kpc}$ (and for the outer Galaxy). In addition, there remains a lack of consensus regarding the number, locations, and nomenclature of large-scale structures near the GC (these are discussed in Section 4.1). Despite this, H I absorption associated with EIG features was successfully used as a distance indicator, allowing for constraints on the line-of-sight distance for over $80 \%$ of the sample of $\mathrm{H}$ II regions investigated, or over $60 \%$ of all known $\mathrm{H}$ II regions with systemic velocities in $|l|<10^{\circ}$.

Over $67 \%$ of the $\mathrm{H}$ II regions demonstrate $\mathrm{HI}$ absorption associated with the Near $3 \mathrm{kpc}$ Arm (see Table 2) and therefore must be located at line-of-sight distances of at least $\sim 5 \mathrm{kpc}$. 
A further $16 \mathrm{H}$ II regions show absorption associated with EIG features assumed to lie further along the line of sight than the Near $3 \mathrm{kpc}$ Arm, therefore over $78 \%$ of the sample $\mathrm{H}$ II regions are located at $D_{\text {los }} \gtrsim 5 \mathrm{kpc}$. This is in keeping with the work of Lang et al. (2010) who find $\sim 90 \%$ of their sample of 40 EIG continuum sources must be located at least as far as the Near $3 \mathrm{kpc}$ Arm.

Of the $151 \mathrm{H}$ II regions investigated, $54 \mathrm{H}$ II regions display absorption from EIG features assumed to be on the far side of the GC (the $+135 \mathrm{~km} \mathrm{~s}^{-1}$ Expanding Arm, Bania's Clump 1, or Far $3 \mathrm{kpc}$ Arm). Consequently, these $\mathrm{H}$ iI regions must be located at $D_{\text {los }} \gtrsim 8.5 \mathrm{kpc}$.

After successfully resolving the near/far kinematic distance ambiguity, line-of-sight distances were calculated for $31 \mathrm{H} \mathrm{II}$ regions. These distances suggest locations for the $\mathrm{H}$ II regions in known Galactic structures including the Norma, Sagittarius, and Perseus spiral arms (see Figure 7). The seven H II regions beyond the solar circle are among the most distant Galactic $\mathrm{H}$ II regions known to exist and could be crucial to tracing the Scutum-Centaurus Arm; where identification of star formation with molecular tracers is extremely difficult (Dame \& Thaddeus 2011). Errors on these line-of-sight distances are often largedue to the uncertainty of non-circular streaming motions, and differences in Galactic rotation models-but the near/far KDAR remains both valid and significant.

Using a summary of EIG structures, and the known $l v$ distribution of $\mathrm{CO}$, we construct a "crayon diagram" with which to investigate the distribution of $\mathrm{H}_{\mathrm{I}}$ absorption in the EIG (Figure 2 and Section 4). In Section 5, we find cold H i clouds, signified by $\mathrm{H}$ I absorption, associated with the Near $3 \mathrm{kpc}$ Arm, Connecting Arm, Bania's Clump 1, Tilted Disk, and Far 3 kpc Arm. There was minimal $\mathrm{H}$ I absorption associated with either the Looping Ridge or the $+135 \mathrm{~km} \mathrm{~s}^{-1}$ Expanding Arm. The large amount of $\mathrm{H}$ I absorption associated with each of the $3 \mathrm{kpc}$ Arms presented an opportunity to fit a model to the $l v$ locus of each arm (Section 5.1). We find a linear fit (in $l v$ space) that is consistent with the findings of Dame \& Thaddeus (2008), who used CO to trace the arms.

The $l v$ distribution of the RRL velocities of the $151 \mathrm{HII}$ regions was investigated in Section 6. Like the $\mathrm{H}_{\mathrm{I}}$ absorption distribution, the systemic velocities of the H II regions trace Galactic structures including spiral arms, features located near the GC, and possibly the end of the bar. While most $\mathrm{H}$ II regions posses RRL velocity components allowed by circular Galactic rotation (suggestive of a location outside the EIG), smaller numbers of $\mathrm{H}$ II regions are found to be associated with the H I Tilted Disk, Near $3 \mathrm{kpc}$ Arm, $+135 \mathrm{~km} \mathrm{~s}^{-1}$ Expanding Arm, Bania's Clump 1, Connecting Arm, and Far 3 kpc Arm. Using the RRL velocity and $\mathrm{H}_{\mathrm{I}}$ absorption spectrum of each H II region, we were also able to constrain the $D_{\text {los }}$ for a further sample of $\mathrm{H}$ II regions using only EIG features as a distance indicator.

This research has made use of NASA's Astrophysics Data System, the NASA/IPAC Extragalactic Database (NED), and the SIMBAD database.

\section{APPENDIX}

\section{DISCUSSION OF INDIVIDUAL H II REGIONS}

G350.004+00.438. The H I absorption spectrum does not give a clear indication of any absorption associated with any EIG feature. At this longitude, the velocity range of the Far $3 \mathrm{kpc}$
Arm is not clearly distinct from velocities expected by normal circular rotation.

G350.129+00.088. The H I absorption spectra clearly demonstrates absorption either side of the velocities expected by an association with the Near $3 \mathrm{kpc}$ Arm. Quireza et al. (2006) place the $\mathrm{H}$ II region at a line-of-sight distance of $6.2 \mathrm{kpc}$ (i.e., on the near side of the GC).

G350.177+00.017. Evidence of absorption in the Far $3 \mathrm{kpc}$ Arms suggests a far KDAR. As with G350.330+00.157 (below), the calculated $R_{\mathrm{Gal}}$ and $D_{\mathrm{los}}$ for the region are outside the bounds of the McClure-Griffiths \& Dickey (2007) rotation model.

$G 350.330+00.157$. While the $\mathrm{H}$ I absorption spectrum suffers from emission fluctuations around the RRL velocity $\left(\sim-60 \mathrm{~km} \mathrm{~s}^{-1}\right)$, there is evidence of absorption associated with the Near $3 \mathrm{kpc}$ Arm. Assuming a far-side KDAR, the calculated $R_{\mathrm{Gal}}$ and $D_{\text {los }}$ are outside the bounds of the McClure-Griffiths \& Dickey (2007) rotation model.

G350.335+00.107. Evidence of $\mathrm{H}$ I absorption is seen either side of the velocities associated with the Near $3 \mathrm{kpc}$ Arm (see G350.129+00.088 above), and is therefore located at least as far as the Near $3 \mathrm{kpc}$ Arm along the line of sight.

G350.524+00.960. G350.524+00.960 does not demonstrate any $\mathrm{H}_{\mathrm{I}}$ absorption outside the velocities expected by normal circular rotation. If the near kinematic distance is therefore assumed, the $\mathrm{H}$ II region has a calculated $D_{\text {los }} \approx 1.9 \mathrm{kpc}$.

G350.769-00.075. The $\mathrm{HI}$ absorption spectrum of G350.769-00.075 does not give conclusive evidence for either a near, nor far, KDAR.

G350.813-00.019. As the $\mathrm{HI}_{\mathrm{I}}$ absorption spectrum of G350.813-00.019 demonstrates absorption in velocities associated with the Far $3 \mathrm{kpc}$ Arm, the $\mathrm{H}$ II region must be on the far side of the GC. The positive (small) RRL velocity then locates the $\mathrm{H}$ II region at a line-of-sight distance beyond (but close to) the solar circle.

G350.996-00.557. Strong absorption is seen in the Far $3 \mathrm{kpc}$ Arm, but not in the Near $3 \mathrm{kpc}$ Arm. The RRL velocity suggests a location within the Far $3 \mathrm{kpc}$ Arm.

G351.028+00.155. The $\mathrm{HI}_{\mathrm{I}}$ absorption spectrum of G351.028+00.155 demonstrates significant absorption at velocities corresponding to both the Near and Far $3 \mathrm{kpc}$ Arms, and therefore must be located at least as far as the Far $3 \mathrm{kpc}$ Arm along the line of sight. The positive RRL velocity then requires that G351.028+00.155 is located in the outer Galaxy.

G351.047-00.322. The $\mathrm{HI}_{\mathrm{I}}$ absorption spectrum of G351.047-00.322 does not give conclusive evidence for either a near or far KDAR.

G351.192+00.708. H I absorption is evident in circular rotation allowed velocities only. If the near kinematic distance is therefore assumed, the $\mathrm{H}$ II region has a calculated $D_{\operatorname{los}} \approx$ $0.3 \mathrm{kpc}$. Moisés et al. (2011) assume the near kinematic distance, however Quireza et al. (2006) place the H II region at a line-of-sight distance of $17.1 \mathrm{kpc}$.

G351.201+00.483. The $\mathrm{H}_{\mathrm{I}}$ absorption spectrum of G351.201+00.483 does not give conclusive evidence for either a near, nor far, KDAR. Quireza et al. (2006) place the object at $1.4 \mathrm{kpc}$, at the near kinematic location.

G351.358+00.666. Quireza et al. (2006) assume a near KDAR for G351.358+00.666, but $\mathrm{H}$ I absorption associated with velocities expected of the Far $3 \mathrm{kpc}$ Arm suggest that the H II region is located at the far kinematic location.

G351.359+01.014. The $\mathrm{HI}$ absorption spectrum of G351.359+0.1014 does not give conclusive evidence for either a near or far KDAR. 
G351.467-00.462. Quireza et al. (2006) give G351.46700.462 a near-side KDAR, but the $\mathrm{H}$ I absorption spectrum from this paper does not give conclusive evidence for a KDAR.

G351.601-00.348. The RRL velocity for this H II region (-91.8 $\mathrm{km} \mathrm{s}^{-1}$; Lockman 1989) is associated with the Near $3 \mathrm{kpc}$ Arm. Green \& McClure-Griffiths (2011) also position a nearby $6.7 \mathrm{GHz}$ maser $(l, b=351.581,-0.353)$ in the Near $3 \mathrm{kpc}$ Arm.

G351.662+00.518. G351.662+00.518 has a near zero RRL velocity $\left(-2.9 \mathrm{~km} \mathrm{~s}^{-1}\right.$; Lockman 1989) which is associated with locations inside the EIG region, near the solar circle, or at a very small line-of-sight distance from the Sun. Absorption at velocities associated with the Near $3 \mathrm{kpc}$ Arm imply a $D_{\text {los }}>5 \mathrm{kpc}$. As there is no $\mathrm{HI}$ absorption associated with other EIG features (only the Far $3 \mathrm{kpc}$ Arm is expected at this longitude), a location within $R_{\mathrm{Gal}} \lesssim 3 \mathrm{kpc}$ is assumed.

$G 351.691+00.669$. No H I absorption falls outside the circular rotation envelope of allowed velocities, suggesting a near KDAR. However, the positive RRL velocity suggests a location in either the EIG or outer Galaxy.

G352.233-00.151. This H II region has an RRL velocity associated with the Near $3 \mathrm{kpc}$ Arm (-88.6 km s${ }^{-1}$, GBTHRDS). Strong absorption in the allowed circular rotation velocities and at velocities associated with the Near $3 \mathrm{kpc}$ Arm, reaffirm the location in the arm.

G352.313-00.440. Evidence of H I absorption in both the Near and Far $3 \mathrm{kpc}$ Arms suggests a far-side KDAR for G351.313-00.440.

G352.398-00.057. Absorption is seen at the expected velocities of the Near $3 \mathrm{kpc}$ Arm, which is also where the RRL velocity for this $\mathrm{H}$ II region lies $\left(-87 \mathrm{~km} \mathrm{~s}^{-1}\right.$; Lockman 1989). Absorption up to $25 \mathrm{~km} \mathrm{~s}^{-1}$ beyond the RRL velocity of an $\mathrm{H}$ II region is not uncommon (Dickey et al. 2003; Jones \& Dickey 2012), therefore it is assumed that the $\mathrm{H}$ II region is located in the Near $3 \mathrm{kpc}$ Arm.

G352.521-00.144. Two RRL velocities have been recorded for G352.521-00.144 (-57.3 and $-38 \mathrm{~km} \mathrm{~s}^{-1}$; GBTHRDS), suggestive of multiple emission sources along the line of sight.

G352.610+00.177. The $\mathrm{H}$ I absorption spectrum for G352.610+00.177 suffers from emission fluctuations. As a result the poor quality spectrum does not give conclusive evidence for a KDAR.

G352.611-00.172. G352.611-00.172 displays strong absorption at $\sim 100 \mathrm{~km} \mathrm{~s}^{-1}$, approximately $20 \mathrm{~km} \mathrm{~s}^{-1}$ beyond the known RRL velocity of the $\mathrm{H}$ II region $\left(-81.9 \mathrm{~km} \mathrm{~s}^{-1}\right.$; Lockman 1989). As with G352.398-00.057 (above), G352.611-00.172 is assumed to lie in the Near $3 \mathrm{kpc}$ Arm. This location, in the Near $3 \mathrm{kpc}$ Arm, is approximately the same as the line-of-sight distance given by Quireza et al. (2006; $6.7 \mathrm{kpc})$.

G352.866-00.199. Evidence of absorption at velocities corresponding to the Near $3 \mathrm{kpc}$ Arm suggest a $D_{\text {los }} \geqslant 5 \mathrm{kpc}$. Green \& McClure-Griffiths (2011) position a nearby $6.7 \mathrm{GHz}$ methanol maser $(l, b=352.855,-0.201)$ at the far kinematic location $\left(D_{\mathrm{los}} \approx 11 \mathrm{kpc}\right)$.

G353.186+00.887. H I absorption is evident in circular rotation allowed velocities only, G353.186+00.887. If the near kinematic location is then assumed, the $\mathrm{H}$ II region has a calculated $D_{\text {los }} \approx 0.9 \mathrm{kpc}$. Quireza et al. (2006) provide a near-side KDAR for this $\mathrm{H}$ II region.

G353.218-00.249. Also the source of a variable maser (Caswell et al. 2010), G353.218-00.249, has a small RRL velocity (-8.3 $\mathrm{km} \mathrm{s}^{-1}$; GBTHRDS) and absorption present at Near 3 kpc Arm, but not Far 3 kpc Arm, velocities. These are evidence for a location near the EIG, and as such $D_{\mathrm{los}} \geqslant 5 \mathrm{kpc}$, $R_{\text {gal }}<3 \mathrm{kpc}$ is assumed.

G353.381-00.114. The $\mathrm{H}$ I absorption spectrum of G353.381-00.114 displays strong $\mathrm{H}_{\mathrm{I}}$ absorption associated with the Near $3 \mathrm{kpc}$ Arm, suggesting that the H II region must lie behind the feature.

G353.398-00.391. H I absorption is evident in circular rotation allowed velocities only; if, therefore, a near-side KDAR is assumed, the calculated $D_{\text {los }} \approx 5.2 \mathrm{kpc}$.

G353.557-00.014. H I absorption is present at velocities corresponding to both $3 \mathrm{kpc}$ Arms, suggestive of a far-side KDAR. However, like the $\mathrm{H}$ II regions G350.330+00.157 and G350.177+00.017, the calculated $R_{\mathrm{Gal}}$ and $D_{\mathrm{los}}$ fall outside the boundaries of the McClure-Griffiths \& Dickey (2007) rotation model.

G354.200-00.050. Strong absorption is centered at velocities to the negative side of those expected for the Near $3 \mathrm{kpc}$ Arm (see G352.611-00.172 and G352.398-0.057 above). Green \& McClure-Griffiths (2011) were unable to determine a KDAR for a nearby $6.7 \mathrm{GHz}$ methanol maser $(l, b=354.206,-0.038)$. Due to the $\mathrm{H}$ I absorption associated with the Near $3 \mathrm{kpc}$ Arm $D_{\text {los }} \geqslant 5 \mathrm{kpc}$ is assumed.

G354.418+0.036. The H I absorption spectrum of G354.418+ 0.036 does not give conclusive evidence for either a near or far KDAR.

G354.486+00.085. Caswell et al. (2010) place a nearby $6.7 \mathrm{GHz}$ methanol maser $(l, b=354.496,0.083)$ in the Far $3 \mathrm{kpc}$ Arm. The RRL velocity of the $\mathrm{H}_{\mathrm{II}}$ region $\left(15.8 \mathrm{~km} \mathrm{~s}^{-1}\right.$; Lockman 1989) is slightly smaller than that expected for the Far $3 \mathrm{kpc}$ Arm, but the absorption indicates the $\mathrm{H}$ II region must be located at least as far along the line of sight as the feature. Due to the positive RRL velocity, we assume that G354.486+00.085 is located beyond the solar circle (see Table 4).

G354.588+00.007. A line of sight along the longitude of 354.588 intersects the Near and Far $3 \mathrm{kpc}$ Arms as well as the assumed position of Bania's Clump 1. The H I absorption spectrum of G354.588+00.007 does not give conclusive evidence for either a near, nor far, KDAR; but absorption associated with the Near $3 \mathrm{kpc}$ Arm suggests $D_{\text {los }} \geqslant 5 \mathrm{kpc}$.

G354.610+00.484. Significant H I absorption is present before and after the velocities expected of the Near $3 \mathrm{kpc}$ Arm, as well as at Far $3 \mathrm{kpc}$ Arm velocities. A known strong $6.7 \mathrm{GHz}$ methanol maser is also in the region (Caswell et al. 2010), with a velocity equivalent to the RRL velocity (maser velocity: $-23 \mathrm{~km} \mathrm{~s}^{-1}$; RRL velocity: $-23.4 \mathrm{~km} \mathrm{~s}^{-1}$ (GBTHRDS)). Green \& McClure-Griffiths (2011) suggest a poor quality nearside KDAR for the associated maser, but a far kinematic distance is assumed here.

G354.664+00.470. H I absorption is evident in circular rotation allowed velocities only, assuming a near-side KDAR the calculated $D_{\mathrm{los}} \approx 4.5 \mathrm{kpc}$.

G354.665+00.247. No absorption is seen at the RRL velocity of the $\mathrm{H}$ II region ( $97.8 \mathrm{~km} \mathrm{~s}^{-1}$; Lockman 1989), nor at velocities corresponding to the Near $3 \mathrm{kpc}$ Arm. However, significant absorption is seen at $\sim 70 \mathrm{~km} \mathrm{~s}^{-1}$, possibly associated with Bania's Clump 1. No KDAR is given here, however the high RRL velocity is suggestive of a location in the EIG (Caswell et al. 2010).

G354.717+00.293. As with G354.665+00.247, the high RRL velocity of G354.717+00.293 suggests a location in the EIG. The H I absorption spectrum suffers from emission fluctuations at the RRL velocity (95.3 $\mathrm{km} \mathrm{s}^{-1}$; GBTHRDS) and no absorption is present at Near $3 \mathrm{kpc}$ Arm velocities. At least two masers 
with high velocities $\left(\sim 100 \mathrm{~km} \mathrm{~s}^{-1}\right)$ are known to exist in the area (Caswell et al. 2010).

G354.934+00.327. G354.934+00.327 shares a similar absorption profile to that of G354.717+00.293 and G354.665+ 00.247 , however it does not share a highly non-circular RRL velocity (14 $\mathrm{km} \mathrm{s}^{-1}$, Caswell \& Haynes 1987). Absorption velocities corresponding to all expected EIG features requires the $\mathrm{H}$ II region to be located at least as far along the line of sight as the Far $3 \mathrm{kpc}$ Arm. Due to the positive RRL velocity, the $\mathrm{H}$ II region must then be located in the outer Galaxy, beyond the solar circle along the line of sight.

G354.979-00.528. The $\mathrm{HI}_{\mathrm{I}}$ absorption spectrum of G354.979-00.528 does not give conclusive evidence for either a near or far KDAR.

G355.242+00.096. H I absorption is present at velocities corresponding to the Near $3 \mathrm{kpc}$ Arm on the near side of the $\mathrm{GC}$, and there is evidence of absorption on the far side of the GC due to the Far $3 \mathrm{kpc}$ Arm and $+135 \mathrm{~km} \mathrm{~s}^{-1}$ Expanding Arm. A far-side KDAR is given, but due to the positive RRL velocity, the $\mathrm{H}$ in region must be located beyond the solar circle.

G355.344+00.145. Absorption at the Near $3 \mathrm{kpc}$ Arm, $+135 \mathrm{~km} \mathrm{~s}^{-1}$ Expanding Arm and Far $3 \mathrm{kpc}$ Arm infer that the $\mathrm{H}_{\mathrm{II}}$ region is located beyond the EIG along the line of sight. The positive RRL velocity then places the H II region beyond the solar circle. There are several masers in the region which are assumed to lie within $3 \mathrm{kpc}$ of the GC (see $(l, b)=$ $(355.343,+0.148),(355.344,+0.147)$, and $(355.346,+0.149)$ in Green \& McClure-Griffiths 2011).

G355.532-00.100. This region has four known RRL velocities $\left(3.8,-22.5,-80.6\right.$, and $-41.1 \mathrm{~km} \mathrm{~s}^{-1}$; GBTHRDS), a strong indication that there are several emission sources along the line of sight. Note that the RRL velocity $-80.6 \mathrm{~km} \mathrm{~s}^{-1}$ is associated with velocities expected of the Near $3 \mathrm{kpc}$ Arm. No $\mathrm{KDAR}$ is given.

G355.581+00.288. Three RRL velocities are known toward the $\mathrm{H}$ II region $\left(+108.7,-76.1\right.$, and $+11.7 \mathrm{~km} \mathrm{~s}^{-1}$; GBTHRDS $)$. As with G355.532-00.100 (above), this is an indication of several sources along the line of sight. No KDAR is given, however; the RRL velocity $-76.1 \mathrm{~km} \mathrm{~s}^{-1}$ is associated with the Near $3 \mathrm{kpc}$ Arm and the RRL velocity component $+108.7 \mathrm{~km} \mathrm{~s}^{-1}$ is associated with the $+135 \mathrm{~km} \mathrm{~s}^{-1}$ Expanding Arm.

$G 355.611+00.382$. The near zero RRL velocity $\left(-2.6 \mathrm{~km} \mathrm{~s}^{-1}\right.$; GBTHRDS) is indicative of an EIG location, or a location near the solar circle (either very close or at a great distance from the Sun). Absorption in velocities associated with the Near $3 \mathrm{kpc}$ Arm and $+135 \mathrm{~km} \mathrm{~s}^{-1}$ Expanding Arm, but not at velocities corresponding to far-side EIG features, prompts $D_{\text {los }} \geqslant 8.5 \mathrm{kpc}, R_{\text {gal }}<3 \mathrm{kpc}$ to be given as a distance limit for the HiI region. In contrast, Green \& McClure-Griffiths (2011) presents a far-side KDAR for a $6.7 \mathrm{GHz}$ methanol maser at $l, b=355.666,+0.398$ which has a systemic velocity of $\sim-2 \mathrm{~km} \mathrm{~s}^{-1}$.

G355.696+0.350. Two RRL velocities (3 and $-79.1 \mathrm{~km} \mathrm{~s}^{-1}$; GBTHRDS) suggest multiple emission sources along the line of sight, at least one of which is associated with the Near $3 \mathrm{kpc}$ Arm (-79.1 $\mathrm{km} \mathrm{s}^{-1}$ RRL association). At this longitude, the velocities of the Near $3 \mathrm{kpc}$ Arm and the Looping Ridge (on the far side of the GC) overlap. No KDAR is given.

G355.700-00.100. G355.700-00.100 has an absorption profile and RRL velocity (-76.1 $\mathrm{km} \mathrm{s}^{-1}$; Lockman 1989) suggestive of a location within the Near $3 \mathrm{kpc}$ Arm or Looping Ridge (as the expected velocities of these features overlap at this longitude). No KDAR is given.
G355.734+0.138. There are multiple RRL velocities associated with G355.734+0.138 (10.7 and $-77.4 \mathrm{~km} \mathrm{~s}^{-1}$; GBTHRDS). No KDAR is given, but the RRL velocity component at $-77.4 \mathrm{~km} \mathrm{~s}^{-1}$ is associated with the velocities expected of the Near $3 \mathrm{kpc}$ Arm or Looping Ridge.

G355.801-00.253. The velocity ranges of the Near $3 \mathrm{kpc}$ Arm and Looping Ridge continue to overlap at this longitude. Two RRL velocities are known $\left(-31.5,3.1 \mathrm{~km} \mathrm{~s}^{-1}\right.$; GBTHRDS), suggestive of multiple sources along the line of sight. No KDAR is given.

G356.230+00.066. At this longitude the expected velocities of the Near $3 \mathrm{kpc}$ Arm and Looping Ridge are distinct (see above). However, the $\mathrm{HI}$ absorption spectrum of G356.230+00.066 does not give conclusive evidence for either a near, nor far, KDAR.

G356.235+00.642. Absorption is seen at velocities corresponding to the Near $3 \mathrm{kpc}$ Arm and Looping Ridge. It is assumed that the H II region is located in the $+135 \mathrm{~km} \mathrm{~s}^{-1} \mathrm{Ex}-$ panding Arm (due to the RRL velocity; $116.3 \mathrm{~km} \mathrm{~s}^{-1}$; Lockman 1989). This is supported by absorption at velocities corresponding to the Looping Ridge (on the far side of the GC, but closer to the GC than the $+135 \mathrm{~km} \mathrm{~s}^{-1}$ Expanding Arm).

G356.307-00.210. A near zero RRL velocity (-4 $\mathrm{km} \mathrm{s}^{-1}$; Lockman 1989) and absorption concurrent with Near 3 kpc Arm velocities suggests $R_{\text {gal }}<3 \mathrm{kpc}$ for this H II region.

G356.470-0.001. The H I absorption spectrum of G356.470-0.001 does not give conclusive evidence for either a near or far KDAR.

G356.560-00.086. The $\mathrm{HI}_{\mathrm{I}}$ absorption spectrum of G356.560-00.086 does not give conclusive evidence for either a near or far KDAR.

G356.650+00.129. H I absorption is present in velocities corresponding to the Near $3 \mathrm{kpc}$ Arm. As such $D_{\text {los }}>5 \mathrm{kpc}$ is assumed.

G357.484-00.036. The H I absorption spectrum suffers from emission fluctuations in the velocity ranges associated with the Near $3 \mathrm{kpc}$ Arm and Looping Ridge. As such the poor quality spectrum does not allow a KDAR to be given for this $\mathrm{H}$ II region.

G357.970-00.169. The $\mathrm{H}_{\mathrm{I}}$ absorption spectrum of G357.970-00.169 displays absorption at velocities associated with the Near $3 \mathrm{kpc}$ Arm. As a result, $D_{\text {los }}>5 \mathrm{kpc}$ is assumed. The small RRL velocity and lack of absorption corresponding to other EIG features suggest a further constraint, $R_{\mathrm{Gal}}<3 \mathrm{kpc}$.

G357.998-00.159. The $\mathrm{H}_{\mathrm{I}}$ absorption spectrum of G357.998-00.159 displays absorption at velocities associated with the Near $3 \mathrm{kpc}$ Arm. As a result, $D_{\mathrm{los}}>5 \mathrm{kpc}$ is assumed. The small RRL velocity, and lack of absorption corresponding to other EIG features suggests a further constraint, $R_{\mathrm{Gal}}<3 \mathrm{kpc}$.

G358.319-00.414. The H I absorption spectrum of G358.319-0.414 does not give conclusive evidence for either a near or far KDAR.

G358.379-00.840. The H I absorption spectrum of G358.379-00.840 does not give conclusive evidence for either a near or far KDAR.

G358.530+00.056. This $\mathrm{H}$ II region has an RRL associated with the Looping Ridge or Tilted Disk (-208.5 $\mathrm{km} \mathrm{s}^{-1}$, GBTHRDS), however the spectrum is of poor quality and no absorption is seen at velocities pertaining to any EIG feature.

G358.552-00.025. This H II region has an RRL associated with the Looping Ridge or Tilted Disk $\left(-208.5 \mathrm{~km} \mathrm{~s}^{-1}\right.$, GBTHRDS), however the spectrum is of poor quality and no absorption is seen at velocities pertaining to EIG features in front of the GC along the line of sight. 
G358.616-00.076. The Hil region has an RRL association with the Tilted Disk or Looping Ridge. The H I absorption spectrum confirms absorption at velocities corresponding to the Near $3 \mathrm{kpc}$ Arm only; further supporting a location in the EIG. Absorption is also seen at velocities either side of the expected velocity range of the Tilted Disk.

G358.623-00.066. Like G358.616-00.076 (above), G358.623-00.066 demonstrates significant absorption associated with the Near $3 \mathrm{kpc}$ Arm and Tilted Disk. The RRL association with the Looping Ridge/Tilted Disk suggests a location in the EIG. Note the bifurcation in the Near $3 \mathrm{kpc}$ Arm absorption profile; see Section 4.

$G 358.633+00.062$. H I absorption is seen at velocities corresponding to the Near $3 \mathrm{kpc}$ Arm and the H I Tilted Disk. The positive RRL velocity suggests either an EIG or near solar circle location: absorption corresponding to near-side EIG features discounts the near kinematic distance; and if the $\mathrm{H}$ II region was located near the solar circle on the far side, there should be evidence of absorption associated with the Far $3 \mathrm{kpc}$ Arm. As a result it is assumed that the $\mathrm{H}_{\mathrm{II}}$ region is located within the EIG, i.e., $R_{\mathrm{Gal}}<3 \mathrm{kpc}, D_{\mathrm{los}}>8.5 \mathrm{kpc}$. Note the bifurcation in the Near $3 \mathrm{kpc}$ Arm absorption profile (see G358.623-00.066 above).

G358.652-00.078, G358.680-00.087, G358.694-00.075, G358.720+00.011, G358.797+00.058, G358.827+00.085, and G359.159-00.038. The $\mathrm{HI}$ absorption profiles of these H II regions are all similar. And all have highly noncircular RRL velocities which correspond to the Tilted Disk-G359.159-00.038 has two known RRL velocities $\left(-182.5\right.$ and $\left.-215.6 \mathrm{~km} \mathrm{~s}^{-1}\right)$. The $\mathrm{H}_{\mathrm{I}}$ absorption spectra suffer from emission fluctuations and are generally of poor quality.

G359.277-00.264. G359.277-00.264 demonstrates no absorption at velocities corresponding to EIG features, a near-side KDAR is given.

G359.432-00.086. G359.432-00.086 has a known RRL velocity associated with the Near $3 \mathrm{kpc}$ Arm. The H I absorption spectrum toward the region demonstrates absorption associated with the Near $3 \mathrm{kpc}$ Arm (and also at $\sim-120 \mathrm{~km} \mathrm{~s}^{-1}$ ).

G359.467-00.172. At this longitude the expected velocity ranges of the Near $3 \mathrm{kpc}$ Arm and Tilted Disk overlap. The $\mathrm{H}$ I absorption spectrum demonstrates absorption at velocities corresponding to the Near $3 \mathrm{kpc}$ Arm, but suffers from emission fluctuations at the overlap. The $\mathrm{H}$ II region has an RRL velocity consistent with either the Tilted Disk or Near $3 \mathrm{kpc}$ Arm.

G000.284-00.478. Absorption is present at velocities corresponding to the Near $3 \mathrm{kpc}$ Arm, but not at the expected velocities of other EIG features. It is assumed that the H II region is located in the EIG, beyond the Near $3 \mathrm{kpc}$ Arm; $R_{\mathrm{Gal}}<3 \mathrm{kpc}$, $D_{\text {los }}>8.5 \mathrm{kpc}$.

G000.361-00.780. G000.361-00.780 demonstrates H I absorption at velocities associated with the Near $3 \mathrm{kpc}$ Arm, but no absorption at other EIG $l v$ features. It is therefore assumed, as with G000.284-00.478 above, that the $\mathrm{H}$ II region is located within $R_{\mathrm{Gal}}<3 \mathrm{kpc}, D_{\text {los }}>8.5 \mathrm{kpc}$.

G000.382+00.107. With two RRL velocities (25.7 and $41.4 \mathrm{~km} \mathrm{~s}^{-1}$; GBTHRDS), the absorption spectrum is likely to have contributions from at least emission two sources along the line of sight. Absorption at the Near $3 \mathrm{kpc}$ Arm and $+135 \mathrm{~km} \mathrm{~s}^{-1}$ Expanding Arm velocities suggests that at least one of the emission sources is located on the far side of the GC. No KDAR is given. Note also that at this longitude, the expected velocity ranges of the $\mathrm{HI}_{\mathrm{I}}$ Tilted Disk and Far $3 \mathrm{kpc}$ Arm are nearly indistinguishable.
G000.510-00.051. H I absorption is present at velocities corresponding to the Near $3 \mathrm{kpc}$ Arm, but not at the velocities of other EIG features. The RRL velocity $\left(45 \mathrm{~km} \mathrm{~s}^{-1}\right.$; Downes et al. 1980) suggests an association with the Far $3 \mathrm{kpc}$ Arm.

G000.572-00.628. The $\mathrm{H}_{\mathrm{I}}$ absorption spectrum of G000.572-00.628 does not give conclusive evidence for either a near, nor far, KDAR.

G000.640+00.623. A far-side KDAR is assumed for G000.640+00.623 due to absorption at velocities corresponding to both $3 \mathrm{kpc}$ Arms (and the Tilted Disk).

G000.729-00.103. G000.729-00.123 has two recorded RRL velocities (105.3 and $83.2 \mathrm{~km} \mathrm{~s}^{-1}$; GBTHRDS), both forbidden by circular Galactic rotation. The region was studied by Downes et al. (1980) who found an RRL velocity of $102 \mathrm{~km} \mathrm{~s}^{-1}$. Caswell \& Haynes (1987) discussed the $\mathrm{H}$ II region as being clearly located near the EIG, but not delineating the outer boundary of the Galactic bar. The GBTHRDS find that of their nine $\mathrm{H}$ II regions associated (in $l v$ space) with the $\mathrm{Nu}$ clear Disk, G000.729-0.103 is the only source that could be located on the redshifted side. The H I spectrum demonstrates statistically significant absorption at velocities corresponding to both $3 \mathrm{kpc}$ Arms, but not for the Nuclear Disk nor $+135 \mathrm{~km} \mathrm{~s}^{-1}$ Expanding Arm. No Hi absorption is present at either of the RRL velocities. No KDAR is given.

$G 000.838+00.189$. The H I absorption spectrum, which is of poor quality due to emission fluctuations, demonstrates absorption consistent with the velocities expected of each of the EIG features (Near $3 \mathrm{kpc}$ Arm, H I Tilted Disk, $+135 \mathrm{~km} \mathrm{~s}^{-1}$ Expanding Arm, and Far $3 \mathrm{kpc}$ Arm). A far-side KDAR is therefore awarded to the $\mathrm{H}$ in region.

G001.125-00.105. Wink et al. (1982) remarked that the noncircular RRL velocity $\left(-19.7 \mathrm{~km} \mathrm{~s}^{-1}\right)$ and $\mathrm{H}_{2} \mathrm{CO}$ at 84 and $123 \mathrm{~km} \mathrm{~s}^{-1}$ was typical of an EIG source; Quireza et al. (2006) also give $D_{\text {los }}=8.5 \mathrm{kpc}$. The $\mathrm{H}$ II region must be located within the EIG, as absorption at EIG features negates the near-side kinematic location and if the $\mathrm{H}$ II region must have a non-realistic $R_{\mathrm{Gal}}>45 \mathrm{kpc}$.

G001.149-00.062. G001.149-00.062 displays absorption at velocities corresponding to both the Near and Far $3 \mathrm{kpc}$ Arms. Assuming a distance of at least the Far $3 \mathrm{kpc}$ Arm, G001.149-00.062 must lie in the outer Galaxy, beyond the solar circle (due to the negative systemic velocity). However, using a flat rotation model for the outer Galaxy, the calculated $D_{\text {los }}$ is unrealistic $(\sim 50 \mathrm{kpc})$. Therefore, the H II region must lie in the EIG region, but behind the Far $3 \mathrm{kpc}$ Arm.

G001.324+00.104. No H I absorption is seen at velocities corresponding to EIG features, suggesting a near KDAR. However, the negative RRL velocity $\left(-12.7 \mathrm{~km} \mathrm{~s}^{-1}\right.$; GBTHRDS) suggests a location in either the EIG or in the outer Galaxy-locations that each would imply absorption by the Near $3 \mathrm{kpc}$ Arm, which is not seen. No KDAR is given.

G001.330+00.088. G001.330+00.088 has a similar H I absorption profile as G001.324+00.104. An EIG location is assumed.

G001.488-0.199. Caswell et al. (2010) assigns a $6.7 \mathrm{GHz}$ methanol maser at the same velocity to $R_{\text {gal }}<3 \mathrm{kpc}$ due to the negative systemic velocity. Absorption at velocities corresponding to the Near $3 \mathrm{kpc}$ Arm supports the $R_{\text {gal }}<3 \mathrm{kpc}$ placement.

G002.009-0.680. $D_{\text {los }}>5 \mathrm{kpc}$ is assumed due to absorption at Near $3 \mathrm{kpc}$ Arm velocities. 
G002.404+0.068. The $\mathrm{HI}$ absorption spectrum of G002.404+0.068 does not give conclusive evidence for either a near or far KDAR.

G002.418-0.611. The H I absorption spectrum of G002.418-0.611 does not give conclusive evidence for either a near or far KDAR.

G002.510-00.028. $D_{\text {los }}>5 \mathrm{kpc}$ is assumed due to absorption at velocities corresponding to the Near $3 \mathrm{kpc}$ Arm.

$G 002.611+00.135$. For a $6.7 \mathrm{GHz}$ methanol maser at the same coordinates, Caswell et al. (2010) discuss that the large positive systemic velocity is most readily attributed to a location within the Galactic bar. Absorption is seen at Near $3 \mathrm{kpc}$ Arm velocities, and at velocities slightly lower than the RRL velocity (102.4 $\mathrm{km} \mathrm{s}^{-1}$; Lockman 1989), but not at $+135 \mathrm{~km} \mathrm{~s}^{-1}$ Expanding Arm velocities; therefore $R_{\text {gal }}<3 \mathrm{kpc}$ is assumed. See Section 6 for a previous discussion of this H II region.

G002.819-00.132. The $\mathrm{HI}$ absorption spectrum of G002.819-00.132 does not give conclusive evidence for either a near, nor far, KDAR.

G002.901-00.006. The negative RRL velocity suggests an EIG or outer Galaxy location. Absorption at Near $3 \mathrm{kpc}$ Arm velocities infers a $D_{\text {los }}>5 \mathrm{kpc}$; but a lack of absorption associated with any other EIG feature does not allow the EIG/outer Galaxy location degeneracy to be resolved. Quireza et al. (2006) give a location in the outer Galaxy.

G002.961-0.053. The H I absorption spectrum is most likely a confusion of multiple $\mathrm{HII}$ regions-there are two RRL velocities (18.1 and $-3.5 \mathrm{~km} \mathrm{~s}^{-1}$; GBTHRDS). No KDAR is given.

G003.270-00.101. Absorption at velocities corresponding to both $3 \mathrm{kpc}$ Arms suggests a kinematic location in, or beyond, the Far $3 \mathrm{kpc}$ Arm. The near zero systemic velocity then suggests a location near the solar circle. Quireza et al. (2006) give a line-of-sight distance to G003.270-00.101 of $14 \mathrm{kpc}$.

G003.342-00.079. Significant absorption is seen at both the Near and Far $3 \mathrm{kpc}$ Arms (and at $\sim 100 \mathrm{~km} \mathrm{~s}^{-1}$ ). Using this absorption as a distance indicator, G003.342-00.079 is given a far KDAR.

G003.439-0.349. G003.439-0.349 is assumed to be located in the Near $3 \mathrm{kpc}$ Arm, due to RRL and maser velocities (GBTHRDS; Caswell et al. 2010), as well as H I absorption, at velocities expected of the Near $3 \mathrm{kpc}$ Arm.

G003.449-0.647. With H I absorption at Near $3 \mathrm{kpc}$ Arm velocities, $D_{\text {los }}>5 \mathrm{kpc}$ is assumed. As the $\mathrm{H}$ II region has a near zero systemic velocity, and no absorption associated with the Far $3 \mathrm{kpc}$ Arm, then $R_{\mathrm{Gal}}<3 \mathrm{kpc}$ should also apply.

G003.655-00.111. Absorption at velocities corresponding to the Near $3 \mathrm{kpc}$ Arm and a near zero RRL velocity $\left(4.6 \mathrm{~km} \mathrm{~s}^{-1}\right.$; Lockman 1989) suggests $R_{\text {gal }}<3 \mathrm{kpc}$.

G003.928-00.116. Evidence of absorption is present at velocities corresponding to both the Near and Far $3 \mathrm{kpc}$ Arms. As a result, G003.928-00.116 is given a far-side KDAR.

G003.949-00.100. The H I absorption spectrum is of poor quality, typical of the diffuse regions of the Lockman et al. (1996) catalog. No KDAR is given, however, the small RRL velocity $\left(6.5 \mathrm{~km} \mathrm{~s}^{-1}\right)$ suggests a possible EIG location.

G004.346+00.115. The $\mathrm{HI}$ absorption spectrum of G004.346+00.115 does not give conclusive evidence for either a near, nor far, KDAR.

G004.412+00.118. Absorption is present at velocities corresponding to the Near and Far $3 \mathrm{kpc}$ Arms. This suggests a location of $R_{\mathrm{gal}}>3 \mathrm{kpc}$ on the far side of the GC; i.e., a far-side KDAR.
G004.527-00.136. Absorption at Near 3 kpc Arm velocities and evidence of absorption at Far $3 \mathrm{kpc}$ Arm velocities suggests a far KDAR.

G004.568-00.118. Absorption at velocities associated with the Near $3 \mathrm{kpc}$ Arm suggest $D_{\mathrm{los}}>5 \mathrm{kpc}$.

G005.193-00.284. Absorption at Near $3 \mathrm{kpc}$ and Connecting Arm velocities suggests $D_{\text {los }}>7 \mathrm{kpc}$; that is, the $\mathrm{H}$ II region must be located behind the Connecting Arm along the line of sight.

G005.479-00.241. Significant absorption is present at velocities associated with both the Near and Far $3 \mathrm{kpc}$ Arms, resulting in a far-side KDAR.

G005.524+00.033. Absorption is present at velocities associated with the Near and Far $3 \mathrm{kpc}$ Arms, suggesting a far-side KDAR.

G005.633+00.240. The $\mathrm{HI}$ absorption spectrum of G005.633+00.240 does not give conclusive evidence for either a near or far KDAR.

G005.889-00.427. Absorption is not seen toward any EIG features, suggesting a near-side KDAR. Downes et al. (1980) also provided a near-side KDAR; however, Quireza et al. (2006) give a line-of-sight distance of $14.5 \mathrm{kpc}$, placing the $\mathrm{H}$ II region on the far side of the GC.

G006.014-00.364. There are two RRL velocities reported for G006.014-00.364 (14.2 and $-31.9 \mathrm{~km} \mathrm{~s}^{-1}$, GBTHRDS) suggesting that there are multiple sources along the line of sight. No KDAR is given.

G006.083-00.117. Absorption at the $3 \sigma_{e}{ }^{-\tau}$ level is seen at velocities associated with the Near and Far $3 \mathrm{kpc}$ Arms, suggesting a far-side KDAR. In addition, absorption at $\sim+135$ is present.

G006.148-00.635. The $\mathrm{H}_{\mathrm{I}}$ absorption spectrum of G006.148-00.635 does not give conclusive evidence for either a near, nor far, KDAR. However, absorption is present at velocities associated with the Near $3 \mathrm{kpc}$ Arm; suggestive of $D_{\text {los }}>5 \mathrm{kpc}$.

G006.160-00.608. H I absorption is present at velocities corresponding to the Connecting Arm, but not the Near $3 \mathrm{kpc}$ Arm (possibly due to emission fluctuations).

G006.225-00.569. The $\mathrm{H}_{\mathrm{I}}$ absorption spectrum of G006.225-00.569 does not give conclusive evidence for either a near, nor far, KDAR. As with G006.160-00.608, absorption is seen at velocities corresponding with the Connecting Arm, but not the Near $3 \mathrm{kpc}$ Arm (which precedes the Connecting Arm along the line of sight).

G006.398-00.474. Absorption is present at velocities corresponding to the Connecting Arm, but not the Near $3 \mathrm{kpc}$ Arm (see above).

G006.553-00.095. Perhaps the most well behaved absorption spectrum in this work; significant absorption is seen in the Near and Far $3 \mathrm{kpc}$ Arms as well as the Connecting Arm, strongly indicative of a far-side KDAR.

G006.565-00.297. Again there is no absorption seen at velocities corresponding to the Near $3 \mathrm{kpc}$ Arm (see G006.160-00.60, G006.225-00.569, and G006.398-00.474 above), but in this case the lack of absorption is probably due to emission fluctuations. Absorption at velocities corresponding to the Connecting and Far $3 \mathrm{kpc}$ Arms suggests a far-side KDAR.

G006.616-00.545. The H I absorption spectrum of G006.616-00.545 does not give conclusive evidence for either a near, nor far, KDAR. 
G007.041+00.176. Significant absorption is present at velocities corresponding to the Near and Far $3 \mathrm{kpc}$ Arms, a far-side $\mathrm{KDAR}$ is given.

G007.176+00.086. The $\mathrm{H}$ I absorption spectrum of G006.616-00.545 does not give conclusive evidence for either a near or far KDAR.

G007.254-00.073. G007.254-00.073 has two known RRL velocities (47 and $17.5 \mathrm{~km} \mathrm{~s}^{-1}$; GBTHRDS), suggesting multiple sources along the line of sight.

G007.266+00.186. The $\mathrm{H}_{\mathrm{I}}$ absorption spectrum of G007.266+00.186 demonstrates absorption at velocities associated with the Near $3 \mathrm{kpc}$ and Connecting Arms, which suggests a location $D_{\text {los }}>7 \mathrm{kpc}$. In addition, a near zero RRL velocity $\left(-4.4 \mathrm{~km} \mathrm{~s}^{-1}\right.$; GBTHRDS), and a lack of absorption at Far $3 \mathrm{kpc}$ Arm velocities suggests $R_{\text {gal }}<3 \mathrm{kpc}$.

G007.299-00.116. The $\mathrm{HI}$ absorption spectrum of G007.299-00.116 does not give conclusive evidence for either a near or far KDAR.

G007.420+00.366. The $\mathrm{H}_{\mathrm{I}}$ absorption spectrum of G007.420+00.366 shows absorption at velocities associated with the Near and Far $3 \mathrm{kpc}$ Arms, as a result the $\mathrm{H}$ II region is given a far-side KDAR.

G007.466-00.279. Absorption is present at velocities associated with the Near $3 \mathrm{kpc}$ Arm, but no further evidence for a KDAR is forthcoming from the $\mathrm{HI}_{\mathrm{I}}$ absorption spectrum.

G007.472+00.060. Significant absorption at velocities corresponding to both the Near and Far $3 \mathrm{kpc}$ Arms strongly suggests a far-side KDAR, however the RRL velocity $\left(-17.8 \mathrm{~km} \mathrm{~s}^{-1}\right.$; Lockman 1989) is indicative of a location in the Near $3 \mathrm{kpc}$ Arm. The $\mathrm{H}$ II region also presents significant absorption at $\sim+135 \mathrm{~km} \mathrm{~s}^{-1}$ (see G006.083-00.117, above).

G007.700-00.079. The H II region has two observed RRL velocities, one of which is associated with the velocity expected of the Connecting Arm. No KDAR is given.

G007.768+00.014. The $\mathrm{H}$ I absorption spectrum of G007.768+0.014 does not give conclusive evidence for either a near or far KDAR.

G007.806-00.621. Evidence of absorption at velocities corresponding to the Near $3 \mathrm{kpc}$ Arm, Connecting Arm implies a distance along the line of sight as least as far as the Connecting Arm.

G008.005-00.484. Absorption is present at velocities consistent with the Near $3 \mathrm{kpc}$ and Connecting Arms, but with no other EIG features. The lower line-of-sight distance limit is therefore $D_{\text {los }}>7 \mathrm{kpc}$.

G008.006-00.156. Evidence of absorption at velocities corresponding to the Near $3 \mathrm{kpc}$ Arm, Connecting Arm and Far $3 \mathrm{kpc}$ Arm strongly implies a far-side KDAR.

G008.094+00.085. The $\mathrm{H}_{\mathrm{I}}$ absorption spectrum of G008.094+00.085 does not give conclusive evidence for either a near or far KDAR.

G008.103+00.340. The H I absorption spectrum of G008.103+00.340 does not give conclusive evidence for either a near or far KDAR. However, absorption is present at velocities corresponding to the Near $3 \mathrm{kpc}$ Arm.

G008.137+00.228. Absorption is not seen toward any EIG features, suggesting a near-side KDAR in agreement with Wink et al. (1982) and Quireza et al. (2006).

G008.362-00.303. The H I absorption spectrum of G008.362-00.303 does not give conclusive evidence for either a near, nor far, KDAR. However, absorption is present at velocities corresponding to the Near $3 \mathrm{kpc}$ Arm.
G008.373-00.352. Absorption is present at velocities consistent with the Near $3 \mathrm{kpc}$ and Connecting Arms, but with no other EIG features.

G008.432-00.276. Evidence of absorption due to both the Near and Far $3 \mathrm{kpc}$ Arms suggests a far-side KDAR

G008.666-00.351. Green \& McClure-Griffiths (2011) and Downes et al. (1980) both give a near-side KDAR for the H II region. A near-side KDAR is also given by this work-note that at this longitude the expected velocity range of the Near $3 \mathrm{kpc}$ Arm overlaps the expected velocities of general circular rotation.

G008.830-00.715. Absorption is not seen toward any EIG features, suggesting a near-side KDAR.

G008.865-00.323. The $\mathrm{HI}$ absorption spectrum of G008.362-00.303 does not give conclusive evidence for either a near or far KDAR.

G009.178+00.043. There $\mathrm{H}_{\mathrm{I}}$ absorption spectrum suffers from emission fluctuations, the $\mathrm{H}$ II region must be located at least as far as the Near $3 \mathrm{kpc}$ Arm along the line of sight.

G009.615+00.198. Kinematic distance analyses can be greatly affected by velocity crowding and a decrease of cold, dense $\mathrm{HI}_{\mathrm{I}}$ in the EIG. For example, Sanna et al. (2009) thoroughly investigated the high-mass star formation region G9.62+0.20 — comprised of several $\mathrm{H}$ II regions - and find from trigonometric parallax that it has a distance of $5.2 \pm 0.6 \mathrm{kpc}$, placing it in the $3 \mathrm{kpc}$ Arm. This distance is at odds with the kinematically determined distances for the region $(0.36$ and $16.4 \mathrm{kpc}$, based on the systemic velocity of the region, $\left.4.1 \mathrm{~km} \mathrm{~s}^{-1}\right)$. Inspection of the H I absorption spectrum of G009.615+00.198 rules out the far kinematic distance as there is no significant absorption at the velocities of far-side EIG features (i.e., the Far $3 \mathrm{kpc}$ Arm).

G009.925-00.745, G009.682+00.206, G009.717-00.832, G009.741+00.842, G009.875-00.749, and G009.982-00.752.

At this longitude the velocities associated with the Near $3 \mathrm{kpc}$ Arm and normal circular rotation overlap. No KDARs are given.

\section{REFERENCES}

Anderson, L. D., \& Bania, T. M. 2009, ApJ, 690, 706

Anderson, L. D., Bania, T. M., Balser, D. S., \& Rood, R. T. 2011, ApJS, 194, 32

Anderson, L. D., Bania, T. M., Balser, D. S., \& Rood, R. T. 2012, ApJ, 754, 62 Baba, J., Saitoh, T. R., \& Wada, K. 2010, PASJ, 62, 1413

Bania, T. M. 1980, ApJ, 242, 95

Bania, T. M., Anderson, L. D., \& Balser, D. S. 2012, ApJ, 759, 96

Bania, T. M., Anderson, L. D., Balser, D. S., \& Rood, R. T. 2010, ApJL, 718, L106

Bania, T. M., \& Lockman, F. J. 1984, ApJS, 54, 513

Bania, T. M., Stark, A. A., \& Heiligman, G. M. 1986, ApJ, 307, 350

Blitz, L., Binney, J., Lo, K. Y., Bally, J., \& Ho, P. T. P. 1993, Natur, 361, 417

Brand, J., \& Blitz, L. 1993, A\&A, 275, 67

Burton, W. B., \& Liszt, H. S. 1983, A\&AS, 52, 63

Caswell, J. L., Fuller, G. A., Green, J. A., et al. 2010, MNRAS, 404, 1029

Caswell, J. L., \& Haynes, R. F. 1987, A\&A, 171, 261

Churchwell, E., Babler, B. L., Meade, M. R., et al. 2009, PASP, 121, 213

Clemens, D. P. 1985, ApJ, 295, 422

Condon, J. J., Cotton, W. D., Greisen, E. W., et al. 1998, AJ, 115, 1693

Dame, T. M., Hartmann, D., \& Thaddeus, P. 2001, ApJ, 547, 792

Dame, T. M., \& Thaddeus, P. 2008, ApJL, 683, L143

Dame, T. M., \& Thaddeus, P. 2011, ApJL, 734, L24

Dickey, J. M., \& Lockman, F. J. 1990, ARA\&A, 28, 215

Dickey, J. M., McClure-Griffiths, N. M., Gaensler, B. M., \& Green, A. J. 2003, ApJ, 585, 801

Downes, D., Wilson, T. L., Bieging, J., \& Wink, J. 1980, A\&AS, 40, 379

Fux, R. 1999, A\&A, 345, 787

Green, J. A., Caswell, J. L., McClure-Griffiths, N. M., et al. 2011, ApJ, 733, 27 Green, J. A., \& McClure-Griffiths, N. M. 2011, MNRAS, 417, 2500 
Green, J. A., McClure-Griffiths, N. M., Caswell, J. L., et al. 2009, ApJL, 696, L156

Gooch, R. 1996, in ASP Conf. Ser. 101, Astronomical Data Analysis Software and Systems V, ed. G. H. Jacoby \& J. Barnes (San Francisco, CA: ASP), 80

Hachisuka, K., Brunthaler, A., Menten, K. M., et al. 2009, ApJ, 696, 1981

Honma, M., Bushimata, T., Choi, Y. K., et al. 2007, PASJ, 59, 889

Jones, C., \& Dickey, J. 2012, ApJ, 753, 62

Kolpak, M. A., Jackson, J. M., Bania, T. M., Clemens, D. P., \& Dickey, J. M. 2003, ApJ, 582, 756

Lang, C. C., Goss, W. M., Cyganowski, C., \& Clubb, K. I. 2010, ApJS, 191,275

Liszt, H. S. 2008, A\&A, 486, 467

Liszt, H. S., \& Burton, W. B. 1980, ApJ, 236, 779

Lockman, F. J. 1989, ApJS, 71, 469

Lockman, F. J., Pisano, D. J., \& Howard, G. J. 1996, ApJ, 472, 173

Marshall, D. J., Fux, R., Robin, A. C., \& Reylé, C. 2008, A\&A, 477, L21

McClure-Griffiths, N. M., \& Dickey, J. M. 2007, ApJ, 671, 427

McClure-Griffiths, N. M., Dickey, J. M., Gaensler, B. M., et al. 2005, ApJS, 158,178

McClure-Griffiths, N. M., Dickey, J. M., Gaensler, B. M., et al. 2012, ApJS, 199, 12
Moisés, A. P., Damineli, A., Figuerêdo, E., et al. 2011, MNRAS, 411, 705

Morris, M., \& Serabyn, E. 1996, ARA\&A, 34, 645

Oort, J. H. 1977, ARA\&A, 15, 295

Paladini, R., Burigana, C., Davies, R. D., et al. 2003, A\&A, 397, 213

Quireza, C., Rood, R. T., Bania, T. M., Balser, D. S., \& Maciel, W. J. 2006, ApJ, 653, 1226

Reid, M. J., Menten, K. M., Zheng, X. W., et al. 2009, ApJ, 700, 137

Rodríguez-Fernández, N. J. 2006, JPhCS, 54, 35

Rodríguez-Fernández, N. J. 2011, MSAIS, 18, 195

Rodriguez-Fernandez, N. J., Combes, F., Martin-Pintado, J., Wilson, T. L., \& Apponi, A. 2006, A\&A, 455, 963

Roy, S. 2003, A\&A, 403, 917

Sanna, A., Reid, M. J., Moscadelli, L., et al. 2009, ApJ, 706, 464

Sewilo, M., Watson, C., Araya, E., et al. 2004, ApJS, 154, 553

Simonson, S. C., \& Madder, G. L. 1973, A\&A, 27, 337

Uchida, K., Morris, M., \& Yusef-Zadeh, F. 1992, AJ, 104, 1533

Urquhart, J. S., Hoare, M. G., Lumsden, S. L., et al. 2012, MNRAS, 420,1656

van Woerden, H., Rougoor, G. W., \& Oort, J. H. 1957, CRAS, 244, 1691

Wink, J. E., Altenhoff, W. J., \& Mezger, P. G. 1982, A\&A, 108, 227 